\title{
ON LADDER HEIGHT DENSITIES AND LAGUERRE SERIES IN THE STUDY OF STOCHASTIC FUNCTIONALS. I. BASIC METHODS AND RESULTS
}

\author{
MICHAEL SCHRÖDER*
}

\begin{abstract}
In this paper we develop methods for reducing the study, the computation, and the construction of stochastic functionals to those of standard concepts such as the moments of the pertinent random variables. Principally, our methods are based on the notion of ladder height densities and their Laguerre expansions, and our results provide a unifying framework for the distinct approaches of Dufresne (2000) and Schröder (2005).
\end{abstract}

Keywords: Laguerre series methods for stochastic functionals; ladder height density

2000 Mathematics Subject Classification: Primary 65C50; 33C45; 60G99

Secondary 33C $90 ; 91 \mathrm{~B} 28$

\section{Introduction}

In this paper we develop methods from orthogonal series into principal ways of analyzing, computing, and constructing stochastic functionals. The main ideas are as follows. Stochastic functionals in their simplest form are expectations,

$$
\mathrm{E}[\rho(Y)]
$$

of images of positive random variables $Y$ under functions $\rho$. Examples of such functionals include the risk-neutral values of contingent claims, with $\rho(Y)$ then the pay-off. It was in fact the observation of Dufresne (2000) that option prices can be constructed as values of probability density functions which has motivated our paper. One of our basic contributions here is to lift this construction to the functional level and to extend Dufresne's approach as follows: expand these densities in Laguerre series, a concept recalled in Section 2; thus obtain a representation of the pertinent functionals as Laguerre series; and reconstruct the coefficients of these Laguerre reduction series in terms of the moments of $Y$.

Alternative approaches to representing stochastic functionals using Laguerre reduction series have been developed in Schröder (2005). The idea of one of them is to expand the pertinent densities as Laguerre series whose coefficients are then in terms of the moments of $Y$, thus representing the functionals as integrals of these series, and obtain Laguerre reduction series on term-by-term integration. A further motivation for this paper is to establish connections between these alternative approaches.

Since both of the described approaches are obviously wrong in general as they stand, the basic idea here is to proceed in the spirit of Schröder (2005) and delineate classes of functionals for which such Laguerre reduction series do exist. We do this by setting up a framework of

Received 23 August 2004; revision received 9 May 2006.

* Postal address: Keplerstrasse 30, D-69469 Weinheim (Bergstrasse), Germany. 
sufficient conditions in terms of the local behaviour of the functions involved, comparing them locally with power mappings or powers of the exponential mapping.

To be able to address both of these aspects and, thus, provide a synthesis of the above two approaches in particular, in Section 4 we first set up how to associate families of ladder height densities with given stochastic functionals. Ladder height density is a concept originally introduced in Dufresne (2000) for option valuation. In Section 3 we extend it into a general context. How Laguerre reduction series for the functionals we have started with are constructed from these families of densities is developed in Section 5. In this way, in Sections 4 and 5 we give a general methodology for associating with a given stochastic functional a family of Laguerre reduction series which represent it.

As an application of these general results, in Section 6 we give a construction of a class of functionals with particularly simple associated Laguerre reduction series families, which contain only the two basic types of reduction series described above. We nevertheless expect this section to be of a particular relevance for applications, for two particular reasons. First, the class of functionals considered is large enough to contain option and power option value functionals. Second, the two Laguerre reduction series associated with each of its functionals address opposite basic needs: the series constructed in the spirit of Dufresne (2000) should enable the construction of these functionals from statistical data, which is a new point of view, and the other series should enable their actual numerical computation.

The power of the above results is demonstrated in Schröder (2006), the companion paper to this one, which studies their applications to exponential functionals of Brownian motion in general, and the valuation of Asian options in particular. The author would be more than gratified if this and the present paper were to furnish starting points of further work on constructive approaches to stochastic functionals. In this sense the author wishes to thank the referee for drawing his attention to the different context and the apparently different purpose to which Laguerre expansions have already been put to work in Anh and Leonenko (1999). There they were found to be instrumental in characterizing limiting fields of rescaled solutions of the heat equation with initial conditions that are nonlinear functionals of random fields with long-range dependence that are not necessarily Gaussian.

\section{Preliminaries and examples}

\subsection{Laguerre series}

In this section we collect pertinent properties of Laguerre polynomials from Lebedev (1972, Section 4) and Sansone (1991). Fixing any real number $\alpha>-1$, for any nonnegative integer $m$ the $m$ th $\alpha$-Laguerre polynomial, $L_{m}^{\alpha}(z)$, is

$$
L_{m}^{\alpha}(z)=\sum_{k=0}^{m} \alpha_{m, k} z^{k}, \quad \text { where } \alpha_{m, k}=\frac{(-1)^{k}}{k !}\left(\begin{array}{l}
m+\alpha \\
m-k
\end{array}\right),
$$

for any complex number $z$. The first three $\alpha$-Laguerre polynomials are $L_{0}^{\alpha}(z)=1, L_{1}^{\alpha}(z)=$ $1+\alpha-z$, and $L_{2}^{\alpha}(z)=\frac{1}{2}(1+\alpha)(2+\alpha)-(2+\alpha) z+\frac{1}{2} z^{2}$. For any positive integer $n$, they satisfy the recurrence relation $(n+1) L_{n+1}^{\alpha}(z)=(2 n+1+\alpha-z) L_{n}^{\alpha}(z)-(n+\alpha) L_{n-1}^{\alpha}(z)$.

Their structural setting is a generalization of the classical Hilbert spaces of square-integrable functions. Let $L_{\alpha}^{2}(0, \infty)$ be the Hilbert space of all complex-valued functions $F$ on the positive reals that are $\alpha$-square integrable, i.e. that satisfy

$$
\|F\|_{\alpha}^{2}=\int_{0}^{\infty} w_{\alpha}(x)|F|^{2}(x) \mathrm{d} x<\infty,
$$


with the weight $w_{\alpha}$ on $(0, \infty)$ given by $w_{\alpha}(x)=x^{\alpha} \exp (-x)$. Then $L_{\alpha}^{2}(0, \infty)$ carries the sesquilinear form $\langle,\rangle_{\alpha}$ given by

$$
\langle F, G\rangle_{\alpha}=\int_{0}^{\infty} w_{\alpha}(x) F(x) \overline{G(x)} \mathrm{d} x,
$$

and an orthogonal basis for $L_{\alpha}^{2}(0, \infty)$ is furnished by the $L_{m}^{\alpha}(z)$, which satisfy $\left\|L_{m}^{\alpha}\right\|_{\alpha}^{2}=$ $\Gamma(m+\alpha+1) / m$ !. By expressing any $F$ in $L_{\alpha}^{2}(0, \infty)$ in this basis, we obtain its $\alpha$-Laguerre series,

$$
F=\sum_{m=0}^{\infty} c_{m} L_{m}^{\alpha}, \quad \text { where } c_{m}=\frac{\left\langle F, L_{m}^{\alpha}\right\rangle_{\alpha}}{\left\langle L_{m}^{\alpha}, L_{m}^{\alpha}\right\rangle_{\alpha}},
$$

for any nonnegative integer $m$. The $c_{m}$ are the $\alpha$-Laguerre coefficients of this series, whose convergence to $F$ is in $\alpha$-mean: $\lim _{M \rightarrow \infty}\left\|F-\sum_{m=0}^{M} c_{m} L_{m}^{\alpha}\right\|_{\alpha}=0$.

\subsection{Growth measures}

Our point of view is that Laguerre expandability is determined by local data. In this section we recall two growth measures proposed in Schröder (2005, Section 2.3) for making this operational and review basic properties derived there, with the Laguerre expansion criterion, Proposition 2.2 below, as a typical result. Our growth measures apply to any complex-valued function $f$ on the positive reals and the idea of them is to describe the behaviour of $f$ at any point $A$ of the extended nonnegative real line, $[0, \infty]$, by comparison with that of power mappings and powers of the exponential function, respectively. Define the exponential growth order, $\delta_{A}(f)$, of $f$ near $A$ as the supremum over all real $\delta$ such that $\lim _{x \rightarrow A} \exp (\delta x) f(x)=0$ :

$$
\delta_{A}(f)=\sup \left\{\delta \in \mathbb{R}: \lim _{x \rightarrow A} \exp (\delta x) f(x)=0\right\} .
$$

The polynomial growth order, $\gamma_{A}(f)$, of $f$ near $A$ is defined as follows: if $A$ is finite it is the supremum over all real $\gamma$ such that $\lim _{x \rightarrow A} f(x) / x^{\gamma}=0$, with the limits taken in the positive reals, i.e.

$$
\gamma_{A}(f)=\sup \left\{\gamma \in \mathbb{R}: \lim _{x \rightarrow A} f(x) / x^{\gamma}=0\right\} ;
$$

otherwise it is defined as $\gamma_{\infty}(f)=\gamma_{0}\left(f^{*}\right)$, where $f^{*}(x)=f(1 / x)$. The respective sets of real numbers $\delta$ and $\gamma$ defining these growth orders may be empty, in which case the suprema are both $-\infty$.

In this paper, the growth orders $\gamma_{0}$ and $\delta_{\infty}$ are of particular relevance. They are illustrated next.

Example 2.1. For any real $c \geq 0$, define the functions $g_{c}(x)=(c-x)^{+}$and $h_{c}(x)=(x-c)^{+}$ on the reals. The growth orders shown in Table 1 can then be checked.

Our growth measures may fail to be addititive but they are log-additive on products.

Proposition 2.1. For any two functions $f, g:(0, \infty) \rightarrow \mathbb{C}$ that are continuous at the point $A$ in $[0, \infty]$, we have $\delta_{A}(f g)=\delta_{A}(f)+\delta_{A}(g)$ if $\left(\delta_{A}(f), \delta_{A}(g)\right) \neq( \pm \infty, \mp \infty)$ and $\gamma_{A}(f g)=$ $\gamma_{A}(f)+\gamma_{A}(g)$ if $\left(\gamma_{A}(f), \gamma_{A}(g)\right) \neq( \pm \infty, \mp \infty)$.

The point of this result, which is Schröder (2005, Proposition 2.1), is to assert that the sums of the growth measures are not just lower bounds for the respective growth measures of the product function, but upper bounds as well. 
TABLE 1: Examples of the growth orders $\gamma_{0}$ and $\delta_{\infty}$.

\begin{tabular}{lcc}
\hline$f$ & $\gamma_{0}(f)$ & $\delta_{\infty}(f)$ \\
\hline$x \mapsto \mathrm{e}^{a x}, a \in \mathbb{R}$ & 0 & $-a$ \\
$x \mapsto x^{a}, a \in \mathbb{R}$ & $a$ & 0 \\
$g_{c}, c>0$ & 0 & $\infty$ \\
$h_{0}$ & 1 & 0 \\
$h_{c}, c>0$ & $\infty$ & 0 \\
\hline
\end{tabular}

The last results can now be applied to give the following Laguerre expansion criterion for a function to have an $\alpha$-Laguerre series in the sense of Section 2.1.

Proposition 2.2. Any continuous function $f:(0, \infty) \rightarrow \mathbb{C}$ is in $L_{\alpha}^{2}(0, \infty)$ if it satisfies $2 \gamma_{0}(|f|)>-(\alpha+1)$ and $2 \delta_{\infty}(|f|)>-1$.

To prove this result, which supersedes Schröder (2005, Proposition 2.2), we have to show that $w_{\alpha}|f|^{2}$ is integrable on the positive reals. Sufficient for this is the following: make sure that near $\infty$ this function decays to 0 at an exponential rate, and that near 0 it can be majorized by a scalar multiple of a power map $x^{\gamma}$ with $\gamma>-1$. Then, using the log-additivity and the results of Example 2.1, respectively transcribe these conditions in terms of $\delta_{\infty}$ and $\gamma_{0}$ to complete the proof.

\section{Ladder height densities}

Ladder height density was proposed in Dufresne (2000). It is a recursively defined concept. Dufresne (2000) used the first and second iterates to represent option values as probability densities, and studied their Laguerre expandability. We consider the general iterates, and, as a first step, in this section study their structural properties.

\subsection{Statement and discussion of main results}

To make precise the ladder height construction of probability density functions, let $g$ be any function on the positive real line which takes its values in the nonnegative reals and which is integrable with total mass 1 . For any nonnegative integer $k$, the $k$ th ladder height density function, $\lambda_{k}(g)$, is then recursively defined by $k$-fold iterated integration, as follows: let $\lambda_{0}(g)=g$ and

$$
\lambda_{k+1}(g)(x)=\frac{1}{M_{1}\left[\lambda_{k}(g)\right]} \int_{x}^{\infty} \lambda_{k}(g)(w) \mathrm{d} w,
$$

for any positive real $x$, if the first moment integral, $M_{1}\left[\lambda_{k}(g)\right]$, is well defined, finite, and positive. The moment functionals $M_{\kappa}$, for any real $\kappa$, are here defined for any measurable function $h$ on the positive reals by

$$
M_{\kappa}[h]=\int_{0}^{\infty} x^{\kappa} h(x) \mathrm{d} x .
$$

Two questions are immediate. First, do probability density functions again result from this construction? Second, how often can we iterate this construction, i.e. which of the $\lambda_{k}(g)$ are well defined? We address these questions in the following basic result, which is proved in Section 3.3. 
Theorem 3.1. For any positive integer $k$, the function $\lambda_{k}(g)$ is well defined if and only if the moments $M_{0}[g], \ldots, M_{k}[g]$ exist and are finite and positive. Then $\lambda_{k}(g)$ is a probability density function and, for any positive real $x$, we have

$$
\lambda_{k}(g)(x)=\frac{k}{M_{k}[g]} \int_{x}^{\infty}(w-x)^{k-1} g(w) \mathrm{d} w .
$$

\subsection{Moments of ladder height densities}

Working in the setting of Section 3.1, in this section we study the $\ell$ th moments of the Section 3.1 ladder height densities $\lambda_{k}(g)$ for nonnegative real $\ell$. More precisely, recalling the moment functionals $M_{\ell}$, we establish the following two results.

Proposition 3.1. If $\lambda_{1}(g), \ldots, \lambda_{k}(g)$ are well defined, we have

$$
M_{\ell}\left[\lambda_{k}(g)\right]=\frac{\Gamma(\ell+1)}{\Gamma(\ell+m+1)} \frac{M_{\ell+m}\left[\lambda_{k-m}(g)\right]}{\prod_{j=1}^{m} M_{1}\left[\lambda_{k-j}(g)\right]}
$$

for any positive integer $m \leq k$, with either both sides finite or both sides infinite.

Corollary 3.1. If $\lambda_{1}(g), \ldots, \lambda_{k}(g)$ are well defined, we have

$$
\prod_{m=0}^{k} M_{1}\left[\lambda_{m}(g)\right]=\frac{M_{k+1}[g]}{(k+1) !} \text { and }\left(\begin{array}{c}
\ell+k \\
\ell
\end{array}\right) M_{\ell}\left[\lambda_{k}(g)\right]=\frac{M_{\ell+k}[g]}{M_{k}[g]}
$$

for any nonnegative integer $\ell$, with either both sides finite or both sides infinite.

Proof of Proposition 3.1. The identity of Proposition 3.1 combines the recursive nature of ladder height densities with a Tonelli-Fubini argument. In fact, in the integrand of the defining integral of the $\ell$ th moment, first express the values of the function $\lambda_{k}(g)$ using their respective defining integrals in terms of $\lambda_{k-1}(g)$ :

$$
M_{\ell}\left[\lambda_{k}(g)\right]=\frac{1}{M_{1}\left[\lambda_{k-1}(g)\right]} \int_{0}^{\infty} \int_{x}^{\infty} x^{\ell} \lambda_{k-1}(g)(y) \mathrm{d} y \mathrm{~d} x .
$$

With $\lambda_{k-1}(g)$ nonnegative and measurable, Tonelli's theorem justifies interchanging the order of integration here, and on performing the inner integration we obtain

$$
M_{\ell}\left[\lambda_{k}(g)\right]=\frac{(\ell+1)^{-1}}{M_{1}\left[\lambda_{k-1}(g)\right]} M_{\ell+1}\left[\lambda_{k-1}(g)\right] .
$$

By induction on $k$, this moment is given by the formula of the proposition with $m$ and $k$ there replaced by $m-1$ and $k-1$, respectively. The formula of the proposition for $M_{\ell}\left[\lambda_{k}(g)\right]$ is then seen to follow on substitution. This completes the induction and, thus, the proof of Proposition 3.1.

Proof of Corollary 3.1. The formulae of Corollary 3.1 are specializations of Proposition 3.1. The first one is obtained by setting $\ell=1$ and $m=k$ there. Substitution of the expression thus obtained for the second denominator in the proposition gives the second formula, and the proof of Corollary 3.1 is complete. 


\subsection{Proof of Theorem 3.1}

The theorem's statements about the moments are established first, by induction. By construction, $\lambda_{1}(g)$ is well defined if and only if $M_{1}[g]$ is finite and positive. Thus, assume $\lambda_{1}(g), \ldots, \lambda_{k}(g)$ to be well defined. Using the second formula of Corollary 3.1, we have

$$
M_{1}\left[\lambda_{k}(g)\right]=\frac{1}{k+1} \frac{M_{k+1}[g]}{M_{1}[g]} .
$$

Inductively, the ladder height densities $\lambda_{1}(g), \ldots, \lambda_{k}(g), \lambda_{k+1}(g)$ are hence well defined if and only if the moments $M_{1}[g], \ldots, M_{k}[g], M_{k+1}[g]$ are finite and positive, as was to be shown. From Proposition 3.1, the mean, $M_{0}\left[\lambda_{n}(g)\right]$, of any such $\lambda_{n}(g)$ is then equal to $M_{1}\left[\lambda_{n}(g)\right] / M_{1}\left[\lambda_{n}(g)\right]=1$. Hence, $\lambda_{n}(g)$ is a probability density.

As a first step in proving the theorem's formula for $\lambda_{k+1}(g)$, an inductive Tonelli-Fubini argument analogous to that used in establishing Proposition 3.1 shows that

$$
\lambda_{k+1}(g)(x)=\frac{1}{\Gamma(k+1) \prod_{j=0}^{k} M_{1}\left[\lambda_{j}(g)\right]} \int_{x}^{\infty}(w-x)^{k} g(w) \mathrm{d} w .
$$

By interpreting the prefactor of the integral using the first formula of Corollary 3.1, we recover the formula of the theorem. The proof of Theorem 3.1 is thus complete.

\section{Ladder height densities and stochastic functionals}

In this section we propose an axiomatic framework for connecting ladder height densities and stochastic functionals, with Theorem 4.1 and Corollary 4.1 as the main results.

\subsection{Stochastic functionals}

Working with any filtered probability space with time set $\left[t_{0}, T\right]$, the stochastic functionals to be considered are expectations of the form

$$
\mathrm{E}\left[\rho(Y) \mid \mathcal{F}_{t}\right], \quad t \in\left[t_{0}, T\right],
$$

conditional on information at time $t$. Here $Y$ is any $\mathcal{F}_{T}$-measurable, positive random variable and $\rho$ is any real-valued function on the positive reals, and they are such that the random variable $\rho(Y)$ is $\mathcal{F}_{T}$-integrable. Moreover, let $\rho$ be a product of two functions, to be specified below, of the form

$$
\rho(y)=\psi(y) \varphi(a, y), \quad y \in(0, \infty) .
$$

Here $\psi$ is a function on the positive reals, $\varphi$ is a function on the product of two copies of the positive reals, and $a$ is a positive real that depends on $\rho$. To fix ideas, it seems instructive to discuss the reciprocal option construction, originating in Dufresne (2000, Section 6).

Example 4.1. Consider any put option with strike price $K>0$ on an asset with positive price process $Z$, and let $Y=1 / Z$ be the reciprocal of $Z$. Assuming risk-neutral valuation to hold for $Z$, with the concepts of Example 2.1 we then have for the option value $\mathrm{E}\left[g_{K}(Z) \mid \mathcal{F}_{t}\right]=$ $\mathrm{E}\left[\rho(Y) \mid \mathcal{F}_{t}\right]$, where $\rho=\psi \varphi$ with $\psi(y)=K / y$ and $\varphi=h_{1 / K}$. Analogous results with the roles of $g$ and $h$ interchanged hold for call options. 


\subsection{Statement and discussion of the main representability results}

In this section we develop an axiomatic framework under which the stochastic functionals of Section 4.1 can be constructed as values of the ladder height density functions described in Section 3. The main results are Theorem 4.1 and its corollary, and a first and fundamental set of conditions for the validity of these representability results is as follows.

Definition 4.1. The triple $(\psi, \varphi, Y)$ is called admissible if the following three conditions are satisfied.

(A1) The function $\psi$ is such that $\mathrm{E}\left[\psi(Y) \mid \mathcal{F}_{t}\right]$ is finite and positive.

(A2) There is a function $\chi$ from the positive reals into themselves such that $\varphi$ is $\chi$-homogeneous, i.e. satisfies $\varphi(\lambda c, \lambda x)=\chi(\lambda) \varphi(c, x)$, for all positive reals $\lambda, c$, and $x$.

(A3) There is a function $L$ from the positive reals into themselves such that, for any real $c>0$, the function $\varphi$ satisfies $\varphi(c, x)=0$ for all $x$ in $(0, L(c)]$.

If the context is clear then, for any real $c>0$, we denote by $\varphi_{c}$ the function $\varphi$ used in Definition 4.1 ; i.e. $\varphi_{c}(x) \equiv \varphi(c, x)$ for any real $x>0$.

While a systematic construction of admissible triples is deferred to Section 4.3 , there is nevertheless the following immediate example.

Example 4.2. We illustrate the axioms for admissibility with examples from option valuation. Here consider the terminal value of the underlying and the strike price as variables. For call options, thus let $\varphi(c, x)=(x-c)^{+}$, whence (A2) and (A3) hold with $\chi(\lambda)=\lambda$ and $L(c)=c$. For put options, however, (A3) fails since $(c-x)^{+}$is never 0 for $x$ in $(0, c)$.

It is instructive to study the immediate consequences of $(\psi, \varphi, Y)$ being admissible. The $\chi$-homogeneity property, condition (A2), gives $\varphi(a, Y)=\chi(a / c) \varphi(c, X)$ for any positive real $c$, with $X=(c / a) Y$. We thus obtain

$$
\mathrm{E}\left[\rho(Y) \mid \mathcal{F}_{t}\right]=\chi\left(\frac{a}{c}\right) \mathrm{E}\left[\psi\left(\frac{a}{c} X\right) \varphi(c, X) \mid \mathcal{F}_{t}\right] .
$$

We record two consequences of this identity in the following two propositions.

First, the positivity and finiteness condition, (A1), implies the following result.

Proposition 4.1. If $f_{X, t}$ is the probability density function of $X$ conditional on information at time $t$, then

$$
g_{c}(x)=\frac{1}{\mathrm{E}\left[\psi(Y) \mid \mathcal{F}_{t}\right]} \psi\left(\frac{a}{c} x\right) f_{X, t}(x), \quad x \in(0, \infty),
$$

defines a probability density function on the positive real line.

Using the vanishing property of $\varphi$, condition (A3), we thus arrive at the following result.

Proposition 4.2. If $(\psi, \varphi, Y)$ is admissible then, for any real $c>0$, we have

$$
\mathrm{E}\left[\rho(Y) \mid \mathcal{F}_{t}\right]=\chi\left(\frac{a}{c}\right) \mathrm{E}\left[\psi(Y) \mid \mathcal{F}_{t}\right] \int_{L(c)}^{\infty} \varphi(c, x) g_{c}(x) \mathrm{d} x .
$$

Our idea is to construct ladder height densities from $g_{c}$, ultimately by fractional integration. Further properties of $\varphi$ and $g_{c}$ are needed for this. We formalize these as follows. 
Definition 4.2. For any nonnegative integer $k$ and any positive real $c$, the pair $\left(\varphi, g_{c}\right)$ is said to permit $k$-fold ladder height densities if the following three properties are satisfied.

(LH1) All ladder height density functions $\lambda_{1}\left(g_{c}\right), \ldots, \lambda_{k}\left(g_{c}\right)$ are well defined, i.e. all moments $M_{1}\left[\lambda_{\ell}\left(g_{c}\right)\right]$ with $0 \leq \ell<k$ are finite and positive.

(LH2) There is a function $L$ from the positive reals into themselves, which we require to coincide with the function $L$ in (A3) if $(\psi, \varphi, Y)$ is admissible, such that for any real $c>0$ the map $\varphi_{c}$ is $k$-fold continuously differentiable on $(L(c), \infty)$.

(LH3) For any real $c>0$, the following limits exist and are finite for all $\ell, 0 \leq \ell<k$ :

$$
\lim _{x \rightarrow \infty}\left(\varphi_{c}^{(\ell)} \lambda_{\ell+1}\left(g_{c}\right)\right)(x) \quad \text { and } \quad \lim _{x \downarrow L(c)}\left(\varphi_{c}^{(\ell)} \lambda_{\ell+1}\left(g_{c}\right)\right)(x) .
$$

Here $\varphi_{c}^{(\ell)}$ denotes any $\ell$ th derivative of the map $\varphi_{c}$.

Under the conditions of Definition 4.2, the representation of Proposition 4.2 can be developed into a representation in terms of ladder height densities as follows.

Theorem 4.1. If $(\psi, \varphi, Y)$ is admissible and the pairs $\left(\varphi, g_{c}\right)$ permit $k$-fold ladder height densities, then we have the following representation for the expectation $\mathrm{E}\left[\rho(Y) \mid \mathcal{F}_{t}\right]$ in terms of ladder height densities $g_{c}=\lambda_{0}\left(g_{c}\right), \lambda_{1}\left(g_{c}\right), \ldots, \lambda_{k}\left(g_{c}\right)$ :

$$
\mathrm{E}\left[\rho(Y) \mid \mathcal{F}_{t}\right]=\sum_{\ell=0}^{k-1} a_{\ell}\left(g_{c}\right)\left[\left(\varphi_{c}^{(\ell)} \lambda_{\ell+1}\left(g_{c}\right)\right)(x)\right]_{x=\infty}^{L(c)}+a_{k-1}\left(g_{c}\right) \int_{L(c)}^{\infty}\left(\varphi_{c}^{(k)} \lambda_{k}\left(g_{c}\right)\right)(x) \mathrm{d} x .
$$

Here the coefficients $a_{\ell}\left(g_{c}\right)$ are

$$
a_{\ell}\left(g_{c}\right)=\chi\left(\frac{a}{c}\right) \frac{(c / a)^{\ell+1}}{(\ell+1) !} \mathrm{E}\left[Y^{\ell+1} \psi(Y) \mid \mathcal{F}_{t}\right]
$$

for any positive real $c$ and any nonnegative integer $\ell$ smaller than $k$.

Corollary 4.1. Let $(\psi, \varphi, Y)$ be admissible and let the pairs $\left(\varphi, g_{c}\right)$ permit $k$-fold ladder height densities. Then we have the representation

$$
\mathrm{E}\left[\rho(Y) \mid \mathcal{F}_{t}\right]=\sum_{\ell=0}^{k-1} a_{\ell}\left(g_{c}\right)\left[\left(\varphi_{c}^{(\ell)} \lambda_{\ell+1}\left(g_{c}\right)\right)(x)\right]_{x=\infty}^{L(c)}+a_{k}\left(g_{c}\right) \varphi_{c}^{(k)}\left(x_{0}\right) \lambda_{k+1}\left(g_{c}\right)(L(c))
$$

if $x \mapsto \varphi_{c}^{(k)}(x)$ is constant on $(L(c), \infty)$ and $\lambda_{k+1}\left(g_{c}\right)$ is well defined.

The proofs of these two results are given in Section 4.4 and ultimately reduce to fractional integration. Making them rigorous, however, requires a fair amount of checking of compatibilities among the normalizing first moments of the various ladder height densities. Immediate from Corollary 4.1, however, is the following result.

Corollary 4.2. Let $(\psi, \varphi, Y)$ be admissible and let the pairs $\left(\varphi, g_{c}\right)$ permit $k$-fold ladder height densities. Then we have the ladder height representation

$$
\mathrm{E}\left[\rho(Y) \mid \mathcal{F}_{t}\right]=a_{k}\left(g_{c}\right) \varphi_{c}^{(k)}\left(x_{0}\right) \lambda_{k+1}\left(g_{c}\right)(L(c))
$$


if $x \mapsto \varphi_{c}^{(k)}(x)$ is constant on $(L(c), \infty)$, if $\lambda_{k+1}\left(g_{c}\right)$ is well defined, and if we have (LH3) in the following sharper form:

$$
\lim _{x \rightarrow \infty}\left(\varphi_{c}^{(\ell)} \lambda_{\ell+1}\left(g_{c}\right)\right)(x)=\lim _{x \downarrow L(c)}\left(\varphi_{c}^{(\ell)} \lambda_{\ell+1}\left(g_{c}\right)\right)(x)=0 \quad \text { for all } \ell \in\{0, \ldots, k-1\} .
$$

Recalling Example 4.1, notice how Corollary 4.2 generalizes a key insight of Dufresne (2000): option values are values of two-fold ladder height densities.

\subsection{Constructing admissible functions}

In this section we present a systematic way of constructing functions $\rho$ that satisfy the conditions of Definition 4.1, Definition 4.2, and, in particular, Theorem 4.1. For this, let $f$ be any function on the positive real line which is equal to 0 on any subinterval $\left(0, x_{0}\right]$. Assume that the function $\chi$ from the positive reals into themselves is multiplicative, i.e. assume that $\chi$ generalizes power mappings in the sense that $\chi(a b)=\chi(a) \chi(b)$ for any positive reals $a$ and $b$. Then we have the following lemma.

Lemma 4.1. In the above setting, the function given on two copies of the positive real line by $\varphi(c, x)=\chi(c) f(x / c)$ satisfies axioms (A2) and (A3) of Definition 4.1.

In fact, we have $\varphi(\lambda c, \lambda x)=\chi(\lambda) \varphi(c, x)$ for any positive reals $\lambda, c$, and $x$, which is (A2). Defining $L(c)=x_{0} c$, we have $\varphi(c, x)=0$ for all $x$ in $(0, L(c)]$ and, hence, (A3).

If, furthermore, $f$ is piecewise $n$-fold continuously differentiable then, for any real $c>0$, $\varphi(c, \cdot)$ inherits this property. We then find the following result for the Section 2.2 growth measures.

Lemma 4.2. In the above setting, assume that $x_{0}$ is not a point of discontinuity of $f$ and its derivatives. For any nonnegative integer $\ell \leq n, L(c)$ is then not a point of discontinuity of the eth derivative of $\varphi(c, \cdot)$, and we have

$$
c \delta_{\infty}\left(\varphi^{(\ell)}(c, \cdot)\right)=\delta_{\infty}\left(f^{(\ell)}\right) \text { and } \gamma_{L(c)}\left(\varphi^{(\ell)}(c, \cdot)\right)=\gamma_{x_{0}}\left(f^{(\ell)}\right) .
$$

To conclude this section we illustrate these concepts by extending Examples 4.1 and 4.2.

Example 4.3. In continuation of Examples 4.1 and 4.2, the factor $\varphi=h_{a}$ there can, for any positive real $a$, be obtained by the above construction. In fact, using the function $f(x)=$ $\left(x-x_{0}\right)^{+}$, we arrive at $\varphi(c, x)=\chi(c)\left(x / c-x_{0}\right)^{+}$. For $x_{0}=1$ and $\chi$ the identity mapping, we thus find that $\varphi(a, x)=(x-a)^{+}=h_{a}(x)$. Regarding growth measures, notice that, for any $c>0$, any $\ell$ th derivative, $\varphi^{(\ell)}(c, \cdot)$, of $\varphi(c, \cdot)$ is nonzero and of at most linear order on $\left(x_{0}, \infty\right)$ if $\ell \leq 1$; otherwise it is identically 0 . For any integer $\ell \geq 2$, we thus obtain

$$
\begin{aligned}
\delta_{\infty}(\varphi(c, \cdot)) & =0, & \delta_{\infty}\left(\varphi^{(1)}(c, \cdot)\right) & =0, & \delta_{\infty}\left(\varphi^{(\ell)}(c, \cdot)\right) & =\infty, \\
\gamma_{L(c)}(\varphi(c, \cdot)) & =\infty, & \gamma_{L(c)}\left(\varphi^{(1)}(c, \cdot)\right) & =-\infty, & \gamma_{L(c)}\left(\varphi^{(\ell)}(c, \cdot)\right) & =\infty,
\end{aligned}
$$

since moreover $\varphi\left(x, x_{0}\right)=0$ and $\varphi^{(1)}\left(c, x_{0}\right)=1$.

\subsection{Preliminaries on moments of the densities $\boldsymbol{g}_{\boldsymbol{c}}$}

As a preliminary to proving the representability results of Section 4.2 , in this section we discuss the $\ell$ th moments, for any real $\ell$, of the probability density $g_{c}$. From Proposition 4.1 the latter is given by

$$
g_{c}(x)=\frac{1}{\mathrm{E}\left[\psi(Y) \mid \mathcal{F}_{t}\right]} \psi\left(\frac{a}{c} x\right) f_{X, t}(x), \quad x \in(0, \infty),
$$


where $X=(c / a) Y$. Recalling the moment functionals $M_{\ell}$ from Section 3.1, the precise results to be established are as follows.

Lemma 4.3. The moments $M_{\ell}\left[g_{c}\right]$ of the probability density function $g_{c}$, assuming their existence and finiteness, are given by

$$
M_{\ell}\left[g_{c}\right]=\left(\frac{c}{a}\right)^{\ell} \frac{\mathrm{E}\left[Y^{\ell} \psi(Y) \mid \mathcal{F}_{t}\right]}{\mathrm{E}\left[\psi(Y) \mid \mathcal{F}_{t}\right]} .
$$

Lemma 4.4. Let $\ell$ be any nonnegative integer. If $\lambda_{\ell}\left(g_{c}\right)$ is well defined then

$$
M_{1}\left[\lambda_{\ell}\left(g_{c}\right)\right]=\frac{1}{\ell+1} \frac{M_{\ell+1}\left[g_{c}\right]}{M_{\ell}\left[g_{c}\right]}=\frac{c / a}{\ell+1} \frac{\mathrm{E}\left[Y^{\ell+1} \psi(Y) \mid \mathcal{F}_{t}\right]}{\mathrm{E}\left[Y^{\ell} \psi(Y) \mid \mathcal{F}_{t}\right]} .
$$

To establish Lemma 4.3, we revert to the definitions, whereby

$$
\mathrm{E}\left[\psi(Y) \mid \mathcal{F}_{t}\right] M_{\ell}\left[g_{c}\right]=\int_{0}^{\infty} x^{\ell} \psi\left(\frac{a}{c} x\right) f_{X, t}(x) \mathrm{d} x=\mathrm{E}\left[X^{\ell} \psi\left(\frac{a}{c} X\right) \mid \mathcal{F}_{t}\right] .
$$

Since $X=(c / a) Y$ by definition, Lemma 4.3 follows. To establish Lemma 4.4, notice that the first identity there follows from the second formula of Corollary 3.1, while the second follows from using Lemma 4.3.

\subsection{Proof of the representability results}

In this section we give proofs of the representability results Theorem 4.1 and Corollary 4.1 .

First, we show how the corollary follows from the theorem if $\left(\partial^{k} \varphi / \partial x^{k}\right)(c, x)=\varphi_{0}$ for any $x$ in $(L(c), \infty)$. This is because the integration term on the right-hand side of the theorem's formula then becomes the value at $L(c)$ of $\lambda_{k+1}\left(g_{c}\right)$, on division by $M_{1}\left[\lambda_{k}\left(g_{c}\right)\right]$, assuming this moment to be finite and positive. The product $a_{k-1}\left(g_{c}\right) M_{1}\left[\lambda_{k}\left(g_{c}\right)\right]$ which results is equal to $a_{k}\left(g_{c}\right)$, using Lemma 4.4 , and the proof of Corollary 4.1 is complete.

We prove the theorem's formula ultimately by successive partial integration starting from the result of Proposition 4.2. The renormalizations in the construction of ladder height densities result in compatibilities that have to be checked, and we give these proofs in two steps.

As a first step, we let $(\varphi, g)$ be any pair which satisfies the three conditions of Definition 4.2 for any real $c>0$ and, setting $b_{\ell}(g)=\prod_{m=0}^{\ell} M_{1}\left[\lambda_{m}(g)\right]$, then prove the identity

$$
\int_{L(c)}^{\infty}\left(\varphi_{c} g\right)(x) \mathrm{d} x=\sum_{\ell=0}^{k-1} b_{\ell}(g)\left[\left(\varphi_{c}^{(\ell)} \lambda_{\ell+1}(g)\right)(x)\right]_{x=\infty}^{L(c)}+b_{k-1}(g) \int_{L(c)}^{\infty}\left(\varphi_{c}^{(k)} \lambda_{k}(g)\right)(x) \mathrm{d} x,
$$

which we will use in step two. We prove the identity by induction on $k$, with the $k=0$ case trivially true. Thus, let $k \geq 1$. Then $\lambda_{1}(g)$ is well defined by (LH1) of Definition 4.2, and can be checked to satisfy $\lambda_{0}(g)=-M_{1}\left[\lambda_{0}(g)\right] \lambda_{1}(g)^{\prime}$. Formal partial integration thus gives

$$
\int_{L(c)}^{\infty}\left(\varphi_{c} \lambda_{0}(g)\right)(x) \mathrm{d} x=M_{1}\left[\lambda_{0}(g)\right]\left[\left(\varphi_{c} \lambda_{1}(g)\right)(x)\right]_{x=\infty}^{L(c)}+M_{1}\left[\lambda_{0}(g)\right] \int_{L(c)}^{\infty}\left(\varphi_{c}^{(1)} \lambda_{1}(g)\right)(x) \mathrm{d} x .
$$

To make this rigorous, notice that $M_{1}\left[\lambda_{0}(g)\right]$ is finite by (LH1), that (LH3) asserts the existence and finiteness of the limits of $\left(\varphi_{c} \lambda_{1}(g)\right)(x)$ as $x$ tends to $L(c)$ and $\infty$, and that (LH2) gives the 
required partial differentiability of $\varphi$. Using $\lambda_{\ell+m}(g)=\lambda_{\ell}\left(\lambda_{m}(g)\right)$, it can now be checked that $\left(\partial \varphi / \partial x, \lambda_{1}(g)\right)$ permits $(k-1)$-fold ladder height densities. Thus, inductively,

$$
\begin{aligned}
\int_{L(c)}^{\infty}\left(\varphi_{c}^{(1)} \lambda_{1}(g)\right)(x) \mathrm{d} x= & \sum_{\ell=0}^{k-2} b_{\ell}\left(\lambda_{1}(g)\right)\left[\left(\varphi_{c}^{(\ell+1)} \lambda_{\ell+1}\left(\lambda_{1}(g)\right)\right)(x)\right]_{x=\infty}^{L(c)} \\
& +b_{k-2}\left(\lambda_{1}(g)\right) \int_{L(c)}^{\infty}\left(\varphi_{c} \lambda_{k}\left(\lambda_{1}(g)\right)\right)(x) \mathrm{d} x .
\end{aligned}
$$

Here first use $\lambda_{\ell+1}\left(\lambda_{1}(g)\right)=\lambda_{\ell+2}(g)$ and $\lambda_{k-1}\left(\lambda_{1}(g)\right)=\lambda_{k}(g)$. Then revert to the definitions, whereby

$$
M_{1}\left[\lambda_{0}(g)\right] b_{\ell}\left(\lambda_{1}(g)\right)=M_{1}\left[\lambda_{0}(g)\right] \prod_{m=0}^{\ell} M_{1}\left[\lambda_{m}\left(\lambda_{1}(g)\right)\right]=b_{\ell+1}(g),
$$

using $\lambda_{m}\left(\lambda_{1}(g)\right)=\lambda_{m+1}(g)$. The induction process for the first step is complete.

As a second step, we compute the coefficients $b_{\ell}(g)$ of the first step for $g=g_{c}$. In fact, successively applying the first formula of Corollary 3.1, and Lemma 4.3, yields

$$
b_{\ell}\left(g_{c}\right)=\frac{M_{\ell+1}\left[g_{c}\right]}{(\ell+1) !}=\frac{(c / a)^{\ell+1}}{(\ell+1) !} \frac{\mathrm{E}\left[Y^{\ell+1} \psi(Y) \mid \mathscr{F}_{t}\right]}{\mathrm{E}\left[\psi(Y) \mid \mathcal{F}_{t}\right]} .
$$

Referring to the Proposition 4.2 representation, the coefficients $a_{\ell}\left(g_{c}\right)$ of the theorem are obtained by multiplying $b_{\ell}\left(g_{c}\right)$ by $\chi(a / c) \mathrm{E}\left[\psi(Y) \mid \mathcal{F}_{t}\right]$. The proof of Theorem 4.1 is thus complete.

\section{Ladder height Laguerre reduction series}

\subsection{Ladder height Laguerre reduction series in an axiomatic setting}

The results of Section 4.2 give a general strategy for studying the stochastic functionals of Section 4.1 using ladder height densities. This section develops the pertinent general results, in particular Theorem 5.1 and Theorem 5.3 and its addenda. They are specialized to the context of Section 4.2 in the next section.

If $g$ is any probability density function on the positive reals which is suitably 'nice' (in a sense to made precise below), our principal idea is to study two problems. As a first step, we study Laguerre expandability of the weighted ladder height density functions $\lambda_{N, \kappa}(g)$, in the sense of Section 2.1. They depend on parameter pairs $\kappa=(\beta, \delta)$ of real numbers, are defined by

$$
\lambda_{N, \kappa}(g)(x)=x^{-\beta} \mathrm{e}^{\delta x} \lambda_{N}(g)(x), \quad x \in(0, \infty),
$$

and are denoted by $\lambda_{N, \beta, \delta}$ if the entries of $\kappa$ are to be stressed. Moreover, let $\phi$ be any suitably nice function on any subinterval $I_{c}=(c, \infty)$ of the positive reals. Defining

$$
\phi_{\kappa}(x) \equiv \phi_{\beta, \delta}(x)=x^{\beta} \mathrm{e}^{-\delta x} \phi(x)
$$

for any $x$ in $I_{c}$, the second problem is then to give conditions under which Laguerre expanding the ladder height density factors of integrals of the form

$$
\int_{I_{c}}\left(\phi_{\kappa} \lambda_{N, \kappa}(g)\right)(x) \mathrm{d} x
$$


leads to convergent series expansions on term-by-term integration. The series that result are in terms of the integrals defined by

$$
I_{k}^{\xi}(g)=\int_{0}^{\infty} x^{\alpha-\beta+k} \mathrm{e}^{(\delta-1) x} \lambda_{N}(g)(x) \mathrm{d} x=\int_{0}^{\infty} x^{k}\left(w_{\alpha} \lambda_{N, \kappa}(g)\right)(x) \mathrm{d} x .
$$

Here $\xi$ is the extension of $\kappa$ to the parameter quadruple $\xi=(N, \alpha, \beta, \delta)$ with any real $\alpha>-1$, and from Section 2.1 we have the weight function $w_{\alpha}(x)=x^{\alpha} \exp (-x)$.

For the first of these Laguerre expansion problems, our results are as follows.

Theorem 5.1. If in the above setting $\lambda_{N}(g)$ is well defined, then, for any $\alpha>-1$ and $\kappa=(\beta, \delta)$ such that $\lambda_{N, \kappa}(g)$ is in $L_{\alpha}^{2}(0, \infty)$, we have the absolutely convergent ladder height Laguerre reduction series

$$
\lambda_{N, \kappa}(g)(x)=\sum_{n=0}^{\infty} b_{n}^{\xi}(g) L_{n}^{\alpha}(x), \quad \xi=(N, \alpha, \beta, \delta),
$$

with $\alpha$-Laguerre coefficients

$$
b_{n}^{\xi}(g)=\sum_{k=0}^{n} \frac{(-1)^{k}}{\Gamma(k+\alpha+1)}\left(\begin{array}{l}
n \\
k
\end{array}\right) I_{k}^{\xi}(g) .
$$

The proof of this result reduces to identifying the Laguerre coefficients $b_{n} \equiv b_{n}^{\xi}$. From Section 2.1, these are given by $b_{n}=\left\langle\lambda_{N, \kappa}(g), L_{n}^{\alpha}\right\rangle_{\alpha} /\left\langle L_{n}^{\alpha}, L_{n}^{\alpha}\right\rangle_{\alpha}$. Linearly expanding the Laguerre polynomials in the first scalar product yields $b_{n}=\sum\left(\alpha_{n, k} /\left\langle L_{n}^{\alpha}, L_{n}^{\alpha}\right\rangle_{\alpha}\right)\left\langle\lambda_{N, \kappa}(g), x^{k}\right\rangle_{\alpha}$. Here $\left\langle\lambda_{N, \kappa}(g), x^{k}\right\rangle_{\alpha}=I_{k}^{\xi}(g)$, on reverting to the integral that defines this scalar product. Making explicit the normalization of the Section 2.1 coefficients $\alpha_{n, k}$, the proof of Theorem 5.1 is complete.

The previous theorem requires us to develop practicable criteria for the Laguerre expandability it requires. For this we single out a class of densities $g$, as follows.

Definition 5.1. A probability density function $g$ is said to be Laguerre admissible if it has the following three properties.

(L0) $g$ is continuous on $(0, \infty)$ with positive values.

(L1) $\delta_{\infty}(g)>0$, i.e. there is a real $\delta>0$ such that $g(x)$ dominates $\exp (-\delta x)$ as $x$ tends to $\infty$, in the sense that $\lim _{x \rightarrow \infty} \exp (\delta x) g(x)$ is 0.

(L2) $\gamma_{0}(g)>-1$, i.e. there is a real $\gamma>-1$ such that $g(x)$ dominates $x^{\gamma}$ as $x$ tends to 0 , in the sense that $\lim _{x \downarrow 0} g(x) / x^{\gamma}$ is 0 .

We give sufficient conditions for the Laguerre expandability of ladder height densities in terms of the densities their construction starts with. Our precise result is provided by the following Laguerre expandability criterion.

Theorem 5.2. Let $g$ be Laguerre admissible and such that the ladder height density $\lambda_{N}(g)$ is well defined. Then, for any reals $\alpha>-1, \beta$, and $\delta$ satisfying the two inequalities

$$
\alpha+2\left(\gamma_{0}(g)+N-\beta\right)>-1 \text { and } 1+2\left(\delta_{\infty}(g)-\delta\right)>0,
$$

the mapping $\lambda_{N, \beta, \delta}(g)$ is in $L_{\alpha}^{2}(0, \infty)$. 
As shown in Section 5.4, this follows from permanence properties of the growth orders $\delta_{\infty}(g)$ and $\gamma_{0}(g)$ under the ladder height construction. These properties are established in Section 5.3 and basically assert that these orders only increase under this process.

For the second of the two Laguerre expansion problems, our results are as follows.

Theorem 5.3. In the above setting, let $g$ be such that $\lambda_{N}(g)$ is well defined. For any $\alpha>-1$ and $\kappa=(\beta, \delta)$ such that $\lambda_{N, \kappa}(g)$ is in $L_{\alpha}^{2}(0, \infty)$ and $\phi_{\kappa}^{2} / w_{\alpha}$ is in $L^{1}\left(I_{c}\right)$, where $I_{c}=(c, \infty)$, we then have the absolutely convergent ladder height Laguerre reduction series

$$
\int_{I_{c}}\left(\phi_{\kappa} \lambda_{N, \kappa}(g)\right)(x) \mathrm{d} x=\sum_{n=0}^{\infty} b_{n}^{\xi}(g) \int_{I_{c}}\left(\phi_{\kappa} L_{n}^{\alpha}\right)(x) \mathrm{d} x
$$

with $\alpha$-Laguerre coefficients

$$
b_{n}^{\xi}(g)=\sum_{k=0}^{n} \frac{(-1)^{k}}{\Gamma(k+\alpha+1)}\left(\begin{array}{l}
n \\
k
\end{array}\right) I_{k}^{\xi}(g),
$$

where $\xi=(N, \alpha, \beta, \delta)$. Its error terms, $R_{N}=\sum_{n=N+1}^{\infty} b_{n}^{\xi}(g) \int_{I}\left(\phi_{\kappa} L_{n}^{\alpha}\right)(x) \mathrm{d} x$, satisfy

$$
R_{N}^{2} \leq\left(\left\|\phi_{\kappa}\right\|_{\alpha}^{2}-\sum_{n=0}^{N} b_{n}^{\xi}(g)^{2}\left\|L_{n}^{\alpha}\right\|_{\alpha}^{2}\right) D_{\xi}
$$

where $D_{\xi}=\int_{I_{c}}\left(\phi_{\kappa}^{2} / w_{\alpha}\right)(x) \mathrm{d} x$ and, recall, $\left\|L_{n}^{\alpha}\right\|_{\alpha}^{2}=\Gamma(n+\alpha+1) / n !$.

Addendum 5.1. Assume that $\phi$ is continuous on $I_{c}=(c, \infty)$, that $g$ is Laguerre admissible, and that $\lambda_{N}(g)$ is well defined. It is then sufficient for $\lambda_{N, \kappa}(g)$ to be in $L_{\alpha}^{2}(0, \infty)$ and $\phi_{\kappa}^{2} / w_{\alpha}$ to be in $L^{1}\left(I_{c}\right)$ that $\phi$ satisfies $2 \gamma_{c}(\phi)>-1$ and that the reals $\beta$ and $\delta$ satisfy the following two inequalities in terms of the growth measures $\gamma_{0}$ and $\delta_{\infty}$ :

$$
\beta<\frac{\alpha+1}{2}+\gamma_{0}(g)+N \text { and } \frac{1}{2}-\delta_{\infty}(\phi)<\delta<\frac{1}{2}+\delta_{\infty}(g) .
$$

Addendum 5.2. We have the following alternative representation:

$$
\left\|\phi_{\kappa}(g)\right\|_{\alpha}^{2}-\sum_{n=0}^{N} b_{n}^{\xi}(g)^{2}\left\|L_{n}^{\alpha}\right\|_{\alpha}^{2}=\sum_{n=N+1}^{\infty} b_{n}^{\xi}(g)^{2}\left\|L_{n}^{\alpha}\right\|_{\alpha}^{2} .
$$

These results incorporate the effects of Theorem 5.2 as a new feature and are analogous to those in Schröder (2005, Section 3.3); they specialize to the latter results for $N=0$. The difficulty in proving them is ultimately to show how convergence in $\alpha$-mean of the Laguerre series of $\phi_{\kappa}(g)$ translates into ordinary convergence on integration. Proofs are now finally given here, in Section 5.5, as an application of Hilbert space theory.

Laguerre expansion of the functions and integrals in Theorem 4.1 is thus reduced to two problems: first, to studying the Laguerre expandability of ladder height densities and, second, to evaluating the above integrals $I(g)$. In general, these integrals define new classes of higher transcendental functions. However, they become expressible in terms of the Section 3.1 moments, $M_{\ell}[g]$, of $g$ when $\delta=1$ can be used, as follows. 
Proposition 5.1. Defining $\xi(1)=\left.(N, \alpha, \beta, \delta)\right|_{\delta=1}$ in the above setting, we have

$$
I_{k}^{\xi(1)}(g)=\frac{M_{N+\delta_{k}}[g]}{M_{N}[g]}\left(\begin{array}{c}
N+\delta_{k} \\
\delta_{k}
\end{array}\right)^{-1}, \quad \text { where } \delta_{k}=\alpha-\beta+k,
$$

with both sides either finite or infinite.

This, as well as a formula for more general integrals $I(g)$, is proved in Section 5.6.

\subsection{Ladder height Laguerre reduction series for stochastic functionals}

In this section we translate the ladder height Laguerre reduction series obtained in Section 5.1 into the stochastic functionals setting of Section 4.1. The main results are Theorem 5.5 and Theorem 5.6 and its addendum. Thus, consider expectations

$$
\mathrm{E}\left[\rho(Y) \mid \mathcal{F}_{t}\right]
$$

conditional on information $\mathscr{F}_{t}$ at time $t$, where the function $\rho$ on the positive reals is such that the image, $\rho(Y)$, of the positive random variable $Y$ is $\mathscr{F}_{T}$-integrable. Moreover, assume that $\rho$ is a product of two functions of positive real arguments,

$$
\rho(y)=\psi(y) \varphi(a, y), \quad y \in(0, \infty),
$$

such that the triple $(\psi, \varphi, Y)$ is admissible in the sense of Definition 4.1 and where $a$ is a positive real that depends on $\rho$. From Proposition 4.1 we then have, for any positive real $c$, the probability density function $g_{c}$ on the positive real line given by

$$
g_{c}(x)=\frac{1}{\mathrm{E}\left[\psi(Y) \mid \mathcal{F}_{t}\right]} \psi\left(\frac{a}{c} x\right) f_{X, t}(x), \quad x \in(0, \infty) .
$$

Here $f_{X, t}$ is the probability density function of the random variable $X=(c / a) Y$ conditional on information at time $t$. It is related to the probability density function $f \equiv f_{Y, t}$, which is conditional on information about $Y$ at time $t$, by $f_{X, t}(x)=(a / c) f((a / c) x)$, for any real $x>0$.

As a first step of the analysis we express the growth measures of $g_{c}$ in terms of those of $\psi$ and the density $f=f_{Y, t}$, in the following two results.

Theorem 5.4. If $(\psi, \varphi, Y)$ is admissible then

$$
\gamma_{0}\left(g_{c}\right)=\frac{a}{c}\left(\gamma_{0}(\psi)+\gamma_{0}(f)\right) \text { and } \delta_{\infty}\left(g_{c}\right)=\frac{a}{c}\left(\delta_{\infty}(\psi)+\delta_{\infty}(f)\right) .
$$

Corollary 5.1. If $g_{c}$ is continuous then any ladder height density $\lambda_{N}\left(g_{c}\right)$ is well defined if

$$
\frac{a}{c}\left(\gamma_{0}(\psi)+\gamma_{0}(f)\right)>-2 \text { and } \delta_{\infty}(\psi)+\delta_{\infty}(f)>0 .
$$

Proof. To reduce the corollary to the theorem, recall from Theorem 3.1 that $\lambda_{N}\left(g_{c}\right)$ is well defined if and only if the moments $M_{1}\left[g_{c}\right], \ldots, M_{N+1}\left[g_{c}\right]$ of $g_{c}$ are finite. For this we have to analyze integrability on the nonnegative real line of the mappings $m_{\ell}(y)=y^{\ell} g_{c}(y)$, which is implied by $\gamma_{0}\left(m_{\ell}\right)>-1$ and $\delta_{\infty}\left(m_{\ell}\right)>0$. Since $\gamma_{0}\left(m_{\ell}\right)=\ell+\gamma_{0}\left(g_{c}\right)$, the theorem translates this condition on $\gamma_{0}$ into $-1<\ell+(a / c)\left(\gamma_{0}(\psi)+\gamma_{0}(f)\right)$ for all $\ell$, whence the $\gamma_{0}$-condition of the corollary follows. On the other hand, $\delta_{\infty}\left(m_{\ell}\right)=\delta_{\infty}\left(g_{c}\right)$. Hence, $\delta_{\infty}\left(m_{\ell}\right)>0$ if and only if $\delta_{\infty}\left(g_{c}\right)>0$. Using the theorem's formula for $\delta_{\infty}\left(g_{c}\right)$, this last condition translates into the $\delta_{\infty}$-condition of the corollary. The proof of Corollary 5.1 is thus complete. 
Proof of Theorem 5.4. We first analyze the behaviour of the growth measures under postmultiplication by positive reals $\mu$. The identity $\exp (\delta x) \psi(\mu x)=\exp ((\delta / \mu) \mu x) \psi(\mu x)$ shows that $\delta_{\infty}(x \mapsto \psi(\mu x))=\mu \delta_{\infty}(\psi)$, and we analogously have $\gamma_{0}(x \mapsto \psi(\mu x))=\mu \gamma_{0}(\psi)$. By the relation $f_{X, t}(x)=\mu f(\mu x)$ with $\mu=a / c$, we similarly have $\delta_{\infty}\left(f_{X, t}\right)=(a / c) \delta_{\infty}(f)$ and $\gamma_{0}\left(f_{X, t}\right)=(a / c) \gamma_{0}(f)$. Applied to the defining relation of $g_{c}$, the log-additivity of $\gamma_{0}$ thus first gives $\gamma_{0}\left(g_{c}\right)=(a / c) \gamma_{0}(\psi)+\gamma_{0}\left(f_{X, t}\right)$ and, hence, on substitution for $\gamma_{0}\left(f_{X, t}\right)$, the first statement of the theorem. For the second statement, notice that log-additivity of $\delta_{\infty}$ similarly implies that $\delta_{\infty}\left(g_{c}\right)=(a / c) \delta_{\infty}(\psi)+\delta_{\infty}\left(f_{X, t}\right)$. On substitution for $\delta_{\infty}\left(f_{X, t}\right)$, the proof of Theorem 5.4 is thus complete.

To address Laguerre expandability of the above functionals, let $(\psi, \varphi, Y)$ be admissible in the sense of Definition 4.1 and assume that $\left(\varphi_{a}, g_{c}\right)$ permits $k$-fold ladder height densities in the sense of Definition 4.2. Then, for any positive real $c$, we have the representation of Theorem 4.1, namely

$$
\mathrm{E}\left[\rho(Y) \mid \mathcal{F}_{t}\right]=\sum_{\ell=0}^{k-1} a_{\ell}\left(g_{c}\right)\left[\left(\varphi_{c}^{(\ell)} \lambda_{\ell+1}\left(g_{c}\right)\right)(x)\right]_{x=\infty}^{L(c)}+a_{k-1}\left(g_{c}\right) \int_{L(c)}^{\infty}\left(\varphi_{c}^{(k)} \lambda_{k}\left(g_{c}\right)\right)(x) \mathrm{d} x,
$$

as well as those of Corollaries 4.1 and 4.2, whose coefficients $a_{\ell}\left(g_{c}\right)$ are, in terms of $\psi$-twisted moments,

$$
a_{\ell}\left(g_{c}\right)=\chi\left(\frac{a}{c}\right) \frac{(c / a)^{\ell+1}}{(\ell+1) !} \mathrm{E}\left[Y^{\ell+1} \psi(Y) \mid \mathcal{F}_{t}\right] .
$$

Laguerre expansion of these functionals thus reduces to two problems: first, Laguerre expansion of the ladder height densities $\lambda_{N}\left(g_{c}\right)$ at, in particular, the point $L(c)$, thus accounting for the Laguerre expansion of the terms in the sum of the above representation; second, application of Laguerre expansions to integrals of such ladder height densities $\lambda_{N}\left(g_{c}\right)$, thus accounting for the integral summand of the above representation.

We now address these two problems in turn, by specializing the results of Section 5.1, assuming that $\delta=1$ there. The first problem, Laguerre expansion of the ladder height densities themselves, is dealt with by the following result.

Theorem 5.5. Let $(\psi, \varphi, Y)$ be admissible, let $g_{c}$ be continuous, and let the reals $\alpha>-1$ and $\beta$ satisfy the following two inequalities:

$$
1<2 \frac{a}{c}\left(\delta_{\infty}(\psi)+\delta_{\infty}(f)\right) \text { and } \max \{2 \beta-(2 N+\alpha+1),-2\}<2 \frac{a}{c}\left(\gamma_{0}(\psi)+\gamma_{0}(f)\right) .
$$

The ladder height density $\lambda_{N}\left(g_{c}\right)$ is then well defined, and we have the absolutely convergent ladder height Laguerre reduction series representation

$$
\lambda_{N}\left(g_{c}\right)(x)=w_{\beta}(x) \sum_{n=0}^{\infty} b_{N, n} L_{n}^{\alpha}(x) .
$$

Here $w_{\beta}$ is the Section 2.1 weight function, given by $w_{\beta}(x)=x^{\beta} \exp (-x)$ on the positive reals, and the $\alpha$-Laguerre coefficients $b_{N, n}=b_{n}^{\xi(1)}\left(g_{c}\right)$ are given by

$$
b_{N, n}=\sum_{k=0}^{n} \frac{(-1)^{k}}{\Gamma(k+\alpha+1)}\left(\begin{array}{l}
n \\
k
\end{array}\right) I_{k}^{\xi(1)}\left(g_{c}\right) \text {. }
$$


Here the $I_{k}^{\xi(1)}$ are the Proposition 5.1 quotients of $\psi$-twisted moments for the parameter quadruple $\xi(1)=\left.(N, \alpha, \beta, \delta)\right|_{\delta=1}$, given by

$$
I_{k}^{\xi(1)}\left(g_{c}\right)=\left(\frac{c}{a}\right)^{\delta_{k}} \frac{\mathrm{E}\left[Y^{N+\delta_{k}} \psi(Y) \mid \mathscr{F}_{t}\right]}{\mathrm{E}\left[Y^{N} \psi(Y) \mid \mathcal{F}_{t}\right]}\left(\begin{array}{c}
N+\delta_{k} \\
\delta_{k}
\end{array}\right)^{-1}
$$

for any reals $\alpha>-1$ and $\beta$ and any nonnegative integers $N$ and $k$, setting $\delta_{k}=\alpha-\beta+k$.

In terms of the same coefficients $b_{N, n}$, the second problem, namely Laguerre expansion of the integrals of the $N$ th derivatives of $\varphi_{c}$ multiplied by the $N$-fold ladder height densities, is taken care of as follows.

Theorem 5.6. Let $(\psi, \varphi, Y)$ be admissible and $g_{c}$ be continuous. Assume both that $\varphi_{c}$ is $N$-fold continuously differentiable on the subinterval $I=(L(c), \infty)$ of the positive real line and that, for reals $\alpha>-1$ and $\beta$, the following four inequalities in terms of the growth measures $\gamma_{c}, \gamma_{0}$, and $\delta_{\infty}$ are satisfied:

$$
\begin{gathered}
-1<\frac{a}{c}\left(\gamma_{0}(\psi)+\gamma_{0}(f)\right), \quad \beta<\frac{\alpha+1}{2}+\frac{a}{c}\left(\gamma_{0}(\psi)+\gamma_{0}(f)\right)+N, \\
2 \gamma_{c}\left(\varphi_{c}^{(N)}\right)>-1, \quad-\delta_{\infty}\left(\varphi_{c}^{(N)}\right)<\frac{1}{2}<\frac{a}{c}\left(\delta_{\infty}(\psi)+\delta_{\infty}(f)\right) .
\end{gathered}
$$

Then the ladder height density $\lambda_{N}\left(g_{c}\right)$ is well defined and, with $w_{\beta}$ the weight function given by $w_{\beta}(x)=x^{\beta} \exp (-x)$ for real $x>0$, we have the absolutely convergent ladder height Laguerre reduction series representation

$$
\int_{I}\left(\varphi_{a}^{(N)} \lambda_{N}\left(g_{c}\right)\right)(x) \mathrm{d} x=\sum_{n=0}^{\infty} b_{N, n} \int_{I}\left(\varphi_{c}^{(N)} w_{\beta} L_{n}^{\alpha}\right)(x) \mathrm{d} x .
$$

Its error terms, $R_{N}=\sum_{n=N+1}^{\infty} b_{N, n} \int_{I}\left(\varphi_{c}^{(N)} w_{\beta} L_{n}^{\alpha}\right)(x) \mathrm{d} x$, satisfy

$$
R_{N}^{2} \leq\left(\left\|\varphi_{c}^{(N)} w_{\beta}\right\|_{\alpha}^{2}-\sum_{n=0}^{N} b_{N, n}^{2}\left\|L_{n}^{\alpha}\right\|_{\alpha}^{2}\right) D_{\alpha}(\beta),
$$

where $D_{\alpha}(\beta)=\int_{I}\left(\left(\varphi_{c}^{(N)} w_{\beta}\right)^{2} / w_{\alpha}\right)(x) \mathrm{d} x$ and, recall, $\left\|L_{n}^{\alpha}\right\|_{\alpha}^{2}=\Gamma(n+\alpha+1) / n !$.

Addendum 5.3. We have the following alternative representation:

$$
\left\|\varphi_{c}^{(N)} w_{\beta}\right\|_{\alpha}^{2}-\sum_{n=0}^{N} b_{N, n}^{2}\left\|L_{n}^{\alpha}\right\|_{\alpha}^{2}=\sum_{n=N+1}^{\infty} b_{N, n}^{2}\left\|L_{n}^{\alpha}\right\|_{\alpha}^{2} .
$$

Proof of the expansion results. The expansions presented in the last two theorems are obtained by specializing Theorem 5.2 and, respectively, Theorem 5.3 plus its addenda, using Theorem 5.4 and Corollary 5.1. We discuss the two together.

First, for $\lambda_{N}\left(g_{c}\right)$ to be well defined it is sufficient, from Corollary 5.1, that the two inequalities $-2<\gamma_{0}\left(g_{c}\right)$ and $0<\delta_{\infty}\left(g_{c}\right)$ are satisfied. For $g_{c}$ to be Laguerre admissible in the sense of Definition 5.1, we have to strengthen the first of them to $-1<\gamma_{0}\left(g_{c}\right)$.

In Theorem 5.5 we have to ensure that $\lambda_{N, \beta, 1}\left(g_{c}\right)$ is additionally in $L_{\alpha}^{2}(0, \infty)$. From Theorem 5.2, for this it is sufficient that the two inequalities $2\left(\gamma_{0}\left(g_{c}\right)+N-\beta\right)>-(\alpha+1)$ 
and $2 \delta_{\infty}\left(g_{c}\right)>1$ are also satisfied. The four inequalities that result can be checked to reduce to the two inequalities of Theorem 5.5 on applying Theorem 5.4. Translating Theorem 5.1 into the present context completes the proof of Theorem 5.5.

We obtain Theorem 5.6 by specializing Theorem 5.3. From Addendum 5.1, for this it is sufficient that $2 \gamma_{c}(\phi)>-1$ and that the two inequalities $\beta<(\alpha+1) / 2+\gamma_{0}\left(g_{c}\right)+N$ and $-\delta_{\infty}(\phi)<\frac{1}{2}<\delta_{\infty}\left(g_{c}\right)$ are satisfied, writing $\phi$ for the $N$ th derivative of $\varphi_{c}$. The five inequalities that result are seen to reduce to the three inequalities of Theorem 5.6 using Theorem 5.4. An application of Theorem 5.3 with $\delta=1$ then yields Theorem 5.6, and Addendum 5.3 follows from translating Addendum 5.2.

It thus remains to establish the explicit formula for the $I\left(g_{c}\right)$ coefficient integrals. Because any $\kappa$ th moment of $g_{c}$ is, from Lemma 4.3, given by

$$
M_{\kappa}\left[g_{c}\right]=\left(\frac{c}{a}\right)^{\kappa} \frac{\mathrm{E}\left[Y^{\kappa} \psi(Y) \mid \mathcal{F}_{t}\right]}{\mathrm{E}\left[\psi(Y) \mid \mathcal{F}_{t}\right]},
$$

this is seen to follow from Proposition 5.1, and the proofs are complete.

In summary, we have thus reduced the computation of expectations $\mathrm{E}\left[\rho(Y) \mid \mathcal{F}_{t}\right]$ to the computation of $\psi$-twisted moments $\mathrm{E}\left[Y^{\kappa} \psi(Y) \mid \mathcal{F}_{t}\right]$. These functions $\psi$ can be thought of as factoring out the non-Laguerre-expandable part of the modified pay-off functions $\rho$. Typical examples of $\psi$ are functions with a pole at 0 of a sufficiently high order. If they are analytic, they then behave like $x^{-\gamma}$, with $\gamma \gg 0$, for small $x$. It is in this sense that $\psi$-twisted moments are close to expectations $\mathrm{E}\left[Y^{m} \mid \mathcal{F}_{t}\right]$ with integral $m$. All these results have been based on those of Section 5.1. The proofs of the latter results are addressed in Sections 5.3 to 5.6.

\subsection{Preliminaries on permanence properties of Laguerre admissibility}

As a preliminary to establishing the Laguerre expandability results of Section 5.1, in this section we study how the Laguerre admissibility of a function, in the sense of Definition 5.1, is transferred to its ladder height densities. We establish the following permanence properties.

Proposition 5.2. Let $g$ be any probability density function with well-defined ladder height densities $\lambda_{1}(g), \ldots, \lambda_{k}(g)$. If $g$ is Laguerre admissible then all of these $\lambda_{\ell}(g)$ are also Laguerre admissible, with growth orders $\delta_{\infty}\left(\lambda_{\ell}(g)\right) \geq \delta_{\infty}(g)$ and $\gamma_{0}\left(\lambda_{\ell}(g)\right) \geq \gamma_{0}(g)+\ell$.

To prove Proposition 5.2, first note that iterated integration as in the Section 3.1 definition of ladder height densities preserves continuity and positivity. Property (L0) of Definition 5.1 thus follows. To establish the remaining two properties, (L1) and (L2), it is sufficient to show that the $\gamma_{0}$-growth behaviour of $g$ and the $\delta_{\infty}$-growth behaviour of $g$ are transferred to the $\lambda_{\ell}(g)$ in the way indicated in the proposition. This we show by induction on $\ell$, with the case $\ell=0$ holding because of the defining relation $\lambda_{0}(g)=g$. To proceed, assume that $\lambda_{\ell+1}(g)$ is well defined, whence

$$
\lambda_{\ell+1}(g)(x)=\frac{1}{M_{1}\left[\lambda_{\ell}(g)\right]} \int_{x}^{\infty} \lambda_{\ell}(g)(x) \mathrm{d} x
$$

follows from Section 3.1. Inductively, if there exists a nonzero real such that $\lambda_{\ell}(g)(x)$ behaves like $D_{\ell} \exp (-a x)$ as $x$ tends to $\infty$, then $\lambda_{\ell+1}(g)(x)$ behaves like $D_{\ell+1} \exp (-a x)$ as $x$ tends to $\infty$, with $D_{\ell+1}=D_{\ell} /\left(a M_{1}\left[\lambda_{\ell}(g)\right]\right)$. Inductively, if there exists an $a>-1$ such that $\lambda_{\ell}(g)(x)$ behaves like $C_{\ell} x^{a}$ as $x$ tends to 0 , then $\lambda_{\ell+1}(g)(x)$ behaves like $C_{\ell+1} x^{a+1}$ as $x$ tends to 0 , with $C_{\ell+1}=C_{\ell} /(a+1)$. This completes the induction and, thus, completes the proof of Proposition 5.2. 


\subsection{Proof of Theorem 5.2}

Recalling the definition of functions in $L_{\alpha}^{2}(0, \infty)$ from Section 2.1, the proof of the Laguerre expandability criterion, Theorem 5.2, reduces to ensuring the finiteness of the integrals

$$
\int_{0}^{\infty} \mathrm{e}^{-x} x^{\alpha}\left(x^{-\beta} \mathrm{e}^{\delta x} \lambda_{N}(g)(x)\right)^{2} \mathrm{~d} x .
$$

With $\lambda_{N}(g)$ being continuous, the existence of the above integrals reduces to studying the integrability of their integrands near 0 and towards $\infty$ in the way made precise in Proposition 2.2. The point is that the permanence properties established in Proposition 5.2 allow the thusobtained sufficient conditions to be written in terms of the growth measures of $g$ itself, as follows.

Integrability near 0 is determined by the $\gamma_{0}$-growth order, $\alpha+2\left(\gamma_{0}\left(\lambda_{N}(g)\right)-\beta\right)$, of the integrand, which has to be greater than -1 . Since $\gamma_{0}\left(\lambda_{N}(g)\right) \geq \gamma_{0}(g)+N$, from Proposition 5.2, this is implied by the condition $\alpha+2\left(\gamma_{0}(g)+N-\beta\right)>-1$ of the theorem.

Integrability towards $\infty$ is determined by the $\delta_{\infty}$-growth order, $1+2\left(\delta_{\infty}\left(\lambda_{N}(g)\right)-\delta\right)$, of the integrand, which has to be positive. Since $\delta_{\infty}\left(\lambda_{N}(g)\right) \geq \delta_{\infty}(g)$, from Proposition 5.2, this is implied by the condition $1+2\left(\delta_{\infty}(g)-\delta\right)>0$ of the theorem. The proof of Theorem 5.2 is thus complete.

\subsection{Proof of Theorem 5.3}

The argument proving the Theorem 5.3 ladder height reduction series and Addendum 5.1 amounts to the argument used to establish Schröder (2005, Theorem 3.2 and Addendum 3.4), which was omitted in that paper. Writing $\lambda \equiv \lambda_{N, \kappa}(g)$ and $\phi \equiv \phi_{\kappa}$ to simplify notation, the idea is as follows. If $\lambda$ is in $L_{\alpha}^{2}(0, \infty)$, let $\lambda=\sum_{n=0}^{\infty} b_{n} L_{n}^{\alpha}(y)$ be its $\alpha$-Laguerre expansion, writing $b_{n} \equiv b_{n}^{\xi}(g)$ for its $n$th $\alpha$-Laguerre coefficient. If interchanging integration with Laguerre series summation is justified, then

$$
\int_{I}(\phi \lambda)(x) \mathrm{d} x=\int_{I}\left(\sum_{n=0}^{\infty} b_{n}\left(\phi L_{n}^{\alpha}\right)(x)\right) \mathrm{d} x=\sum_{n=0}^{\infty} b_{n} \int_{I}\left(\phi L_{n}^{\alpha}\right)(x) \mathrm{d} x,
$$

which is the first assertion of the theorem. Reverting to definitions, a formal computation of the coefficients gives

$$
b_{n}=\frac{\left\langle\lambda, L_{n}^{\alpha}\right\rangle_{\alpha}}{\left\langle L_{n}^{\alpha}, L_{n}^{\alpha}\right\rangle_{\alpha}}=\sum_{k=0}^{n} \frac{\alpha_{n, k}}{\left\langle L_{n}^{\alpha}, L_{n}^{\alpha}\right\rangle_{\alpha}} \int_{I} x^{k}\left(w_{\alpha} \lambda\right)(x) \mathrm{d} x,
$$

where $\alpha_{n, k}$ (from Section 2.1) is the coefficient of $z^{k}$ in $L_{n}^{\alpha}(z)$. The theorem's representation for $b_{n}$ now follows on inspection, recalling that $\left\langle L_{n}^{\alpha}, L_{n}^{\alpha}\right\rangle_{\alpha}=\Gamma(n+\alpha+1) / n$ !.

Establishing the theorem thus reduces to justifying the above term-by-term integration. The difficulty here is to translate the convergence of the series to be proved into the convergence in $\alpha$-mean of the Laguerre series of $\lambda$. Thus, first write

$$
\lambda=\sum_{n=0}^{N} b_{n} L_{n}^{\alpha}+r_{N}, \quad \text { where } r_{N}=\sum_{n=N+1}^{\infty} b_{n} L_{n}^{\alpha} .
$$

Integrating the error terms $r_{N}$, the task is then to establish that

$$
\lim _{N \rightarrow \infty} R_{N}=0, \quad \text { where } R_{N}=\int_{0}^{\infty}\left(\phi r_{N}\right)(y) \mathrm{d} y,
$$


from the convergence of the $r_{N}$ to 0 in $\alpha$-mean. To do so, we proceed as follows. Decompose the integrand of any $R_{N}$ as the product of the functions $\phi / \sqrt{w_{\alpha}}$ and $\sqrt{w_{\alpha}} r_{N}$, majorize the absolute value of the integral by taking the absolute value under the integral sign, and then apply the Hölder inequality of Dunford and Schwartz (1957, Lemma 2, p. 119) with $p=q=2$, to obtain

$$
R_{N}^{2} \leq \int_{0}^{\infty} \frac{\phi^{2}}{w_{\alpha}}(y) \mathrm{d} y \int_{0}^{\infty}\left(w_{\alpha} r_{N}^{2}\right)(y) \mathrm{d} y=D_{\xi}\left\|r_{N}\right\|_{\alpha}^{2} .
$$

Under the conditions of the theorem, this inequality shows how $R_{N}$ goes to 0 with $\left\|r_{N}\right\|_{\alpha}^{2}$ as $N$ tends to $\infty$. At this point we thus have convergence of both the theorem's series and its series representation for $R_{N}$. Absolute convergence of the former series then follows from Riemann's theorem on conditionally convergent series; see Apostol (1974, Theorem 8.33, p. 197).

The norm factor of the last inequality is analyzed using Hilbert space theory along the lines of Katznelson (1976, 5.2 Lemma, p. 28). For this, expand $\left\|r_{N}\right\|_{\alpha}^{2}=\left\langle r_{N}, r_{N}\right\rangle_{\alpha}$ bilinearly on substitution of $r_{N}=\lambda-\left(b_{0} L_{0}^{\alpha}+\cdots+b_{N} L_{N}^{\alpha}\right)$ and use the fact that the $L_{n}^{\alpha}$ are orthogonal under $\langle,\rangle_{\alpha}$, to obtain

$$
\left\|r_{N}\right\|_{\alpha}^{2}=\langle\lambda, \lambda\rangle_{\alpha}-2 \sum_{n=0}^{N} b_{n}\left\langle\lambda, L_{n}^{\alpha}\right\rangle_{\alpha}+\sum_{n=0}^{N} b_{n}^{2}\left\langle L_{n}^{\alpha}, L_{n}^{\alpha}\right\rangle_{\alpha}
$$

Here, substitute for $\left\langle\lambda, L_{n}^{\alpha}\right\rangle_{\alpha}$ from the coefficient formula $b_{n}=\left\langle\lambda, L_{n}^{\alpha}\right\rangle_{\alpha} /\left\langle L_{n}^{\alpha}, L_{n}^{\alpha}\right\rangle_{\alpha}$. In the above inequality for $R_{N}^{2}$ we thus first obtain

$$
R_{N}^{2} \leq D_{\xi}\left(\|\lambda\|_{\alpha}^{2}-\sum_{n=0}^{N} b_{n}^{2}\left\|L_{n}^{\alpha}\right\|_{\alpha}^{2}\right)
$$

which is the inequality for $R_{N}^{2}$ in the theorem. On the other hand, taking the limit as $N$ tends to $\infty$, Parseval's formula results in

$$
0=\lim _{N \rightarrow \infty}\left\|r_{N}\right\|_{\alpha}^{2}=\|\lambda\|_{\alpha}^{2}-\sum_{n=0}^{\infty} b_{n}^{2}\left\|L_{n}^{\alpha}\right\|_{\alpha}^{2}
$$

(see Katznelson (1976, 5.4 Lemma, p. 29)), which gives the assertion of Addendum 5.2.

Establishing the first addendum principally proceeds by reduction to the local properties measured by $\gamma_{0}$ and $\delta_{\infty}$ along the lines of Section 2.2. First, the inequalities

$$
-1<\alpha+2\left(\gamma_{0}(g)+N-\beta\right) \text { and } 0<1+2\left(\delta_{\infty}(g)-\delta\right),
$$

from Theorem 5.2, are sufficient for $\lambda_{N, \kappa}(g)$ to be in $L_{\alpha}^{2}(0, \infty)$. Arguing as for Proposition 2.2, we find the inequalities

$$
-1<2 \gamma_{c}(\phi) \text { and } 0<2\left(\delta_{\infty}(\phi)+\delta\right)-1
$$

to be sufficient for $\phi_{\kappa}^{2} / w_{\alpha}$ to be in $L^{1}(I)$, taking into account the fact that $I=(c, \infty)$ with $c>0$. These four inequalities can be checked to combine into the three given in Addendum 5.1. The proofs of Theorem 5.3 and its two addenda are thus complete. 


\subsection{Computing the integrals $I_{k}^{\xi}(g)$}

In this section, for particular choices of the parameter quadruples $\xi=(N, \alpha, \beta, \delta)$ of Section 5.1, we compute the integrals

$$
I_{k}^{\xi}(g)=\int_{0}^{\infty} y^{\alpha-\beta+k} \mathrm{e}^{(\delta-1) y} \lambda_{N}(g)(y) \mathrm{d} y .
$$

For $\delta=1$ they are equal to the moments $M_{\alpha-\beta+k}\left[\lambda_{N}(g)\right]$, and the second formula of Corollary 3.1 applies to give Proposition 5.1.

To compute them in more general situations, express $\lambda_{N}(g)$ by the integral displayed in Theorem 3.1. Then apply Tonelli's theorem and multiply out the power of $w-x$ in the resulting integrand. Thus, with both sides either finite or infinite,

$$
I_{k}^{\xi}(g)=\frac{N}{M_{N}[g]} \sum_{\ell=0}^{N-1}(-1)^{\ell}\left(\begin{array}{c}
N-1 \\
\ell
\end{array}\right) J_{\ell},
$$

where

$$
J_{\ell}=\int_{0}^{\infty} g(w) \frac{w^{N}}{w^{\ell+1}} \int_{0}^{w} \mathrm{e}^{(\delta-1) y} y^{K_{\ell}} \mathrm{d} y \mathrm{~d} w
$$

with $K_{\ell}=\alpha-\beta+k+\ell$. If $\delta \neq 1$ then the inner integrals of the $J_{\ell}$ are values of the incomplete gamma function; see Erdélyi et al. (1953, Chapter IX) or Press et al. (1992, Section 6.2) for this function. If $\alpha-\beta$ is integral then these are expressible in terms of elementary functions. Assuming that any $K_{\ell}$ is a nonnegative integer, we thus obtain

$$
\begin{aligned}
J_{\ell}= & \frac{K_{\ell} !}{(\delta-1)^{K_{\ell}+1}} M_{N-(\ell+1)}[g] \\
& -\sum_{p=0}^{K_{\ell}} \frac{K_{\ell} !}{\left(K_{\ell}-p\right) !(\delta-1)^{p+1}} \int_{0}^{\infty} \mathrm{e}^{-(\delta-1) w} w^{N+K_{\ell}-(p+1)} g(w) \mathrm{d} w,
\end{aligned}
$$

with both sides either finite or infinite. Here, notice that $\int_{0}^{\infty} w^{a} \mathrm{e}^{b w} g(w) \mathrm{d} w=\mathrm{E}\left[W^{a} \exp (b W)\right]$ if $g$ is the probability density function of any random variable $W$.

\section{Epilogue: functionals with two ladder height reduction series}

In this section we identify a class of stochastic functionals for which our approach in this paper enables a synthesis of the alternative points of view of Dufresne (2000) and Schröder (2005). On the one hand, in Section 6.2 we show how such functionals can be represented as an absolutely convergent series of complex numbers in the spirit of Schröder (2005), which should enable their numerical computation. On the other hand, in Section 6.3 we show how to explicitly construct such functionals as elements of Hilbert spaces, by giving an explicit representation in terms of explicit Hilbert space bases. This type of representation is in the spirit of Dufresne (2000) and, as indicated in Section 6.4, our point of view is that this approach should enable the construction of such functionals from, in particular, statistical data.

\subsection{Basic setting}

We work in the setting introduced in Sections 4.1, 4.2, and 5.1, and thus consider expectations

$$
\mathrm{E}\left[\rho(Y) \mid \mathcal{F}_{t}\right]
$$


conditional on information $\mathcal{F}_{t}$ at any time $t$ in $\left[t_{0}, T\right]$. Here $Y$ is any positive random variable and $\rho$ is any function on the positive reals such that $\rho(Y)$ is $\mathcal{F}_{T}$-integrable and can be factorized as

$$
\rho(x)=\psi(x) \varphi(a, x), \quad x \in(0, \infty),
$$

for functions $\psi$, on the positive reals, and $\varphi$, on two copies of the positive reals, such that the triple $(\psi, \varphi, Y)$ is admissible in the sense of Definition 4.1. Thus, $\mathrm{E}\left[\psi(Y) \mid \widetilde{F}_{t}\right]$ is finite and positive and there are functions $\chi$ and $L$ from the positive reals into themselves such that, for any real $c>0$, we have $\varphi(c,(0, L(c)])=\{0\}$ and $\varphi(\lambda c, \lambda x)=\chi(\lambda) \varphi(c, x)$ for any reals $\lambda, x>0$. While in Section 4.3 we gave a systematic construction of such functions $\varphi$, immediate examples are furnished by the pay-off functions of call options; see Example 4.2 for a discussion.

For convenience, we assume $\psi$ and $f \equiv f_{Y, t}$, the density of $Y$ conditional on information $\mathcal{F}_{t}$ at time $t$, to be continuous functions on the positive reals, and for any real $c>0$ denote by $\varphi_{c}$ the function given by $\varphi_{c}(x) \equiv \varphi(c, x)$, for any real $x>0$. Notice that the growth measures $\gamma_{A}$ and $\delta_{A}$ (see Section 2.2) can then be applied to $\psi$ and $f$, in particular.

\subsection{The first type of reduction series}

Using the concepts and the notation of Section 6.1, in this section we show how ladder height reduction series provide a representation for the stochastic functionals of Section 6.1 in terms of a Laguerre reduction series in the spirit of Schröder (2005). Our precise result about these absolutely convergent series of complex numbers is as follows.

Theorem 6.1. In the setting of Section 6.1, let $c>0, \alpha>-1$, and $\beta$ be any reals such that $\left(w_{\beta} / w_{\alpha}\right) \varphi_{c}$ is in $L_{\alpha}^{2}(0, \infty)$ and the following two inequalities in terms of the growth measures $\gamma_{0}$ and $\delta_{\infty}$ of the functions $\psi$ and $f=f_{Y, t}$ are satisfied:

$$
\beta-\frac{\alpha+1}{2}<\frac{a}{c}\left(\gamma_{0}(\psi)+\gamma_{0}(f)\right) \text { and } \frac{1}{2}<\frac{a}{c}\left(\delta_{\infty}(\psi)+\delta_{\infty}(f)\right) .
$$

Then we have the absolutely convergent Laguerre reduction series representation

$$
\mathrm{E}\left[\rho(Y) \mid \mathcal{F}_{t}\right]=\chi\left(\frac{a}{c}\right) \sum_{n=0}^{\infty} a_{n}\left\langle\frac{w_{\beta}}{w_{\alpha}} \varphi_{c}, L_{n}^{\alpha}\right\rangle_{\alpha},
$$

whose remainder terms, $R_{N}=\chi(a / c) \sum_{n=N}^{\infty} a_{n}\left\langle\left(w_{\beta} / w_{\alpha}\right) \varphi_{c}, L_{n}^{\alpha}\right\rangle_{\alpha}$, satisfy

$$
R_{N}^{2} \leq\left\|\frac{w_{\beta}}{w_{\alpha}} \varphi_{c}\right\|_{\alpha}^{2} \chi^{2}\left(\frac{a}{c}\right) \sum_{n=N}^{\infty} a_{n}^{2}\left\|L_{n}^{\alpha}\right\|_{\alpha}^{2}
$$

recalling that $\left\|L_{n}^{\alpha}\right\|_{\alpha}^{2}=\Gamma(n+\alpha+1) / n !$.

Here, the $w_{\alpha}$ are the weight functions of Section 2.1, given by $w_{\alpha}(x)=x^{\alpha} \exp (-x)$, and the coefficients $a_{n}$ depend on $\alpha, \beta, a$, and $c$ through linear combinations of $\psi$-twisted moments of $Y$, as follows, setting $\delta_{k}=\alpha-\beta+k$ :

$$
a_{n}=\sum_{k=0}^{n} \frac{(-1)^{k}}{\Gamma(k+\alpha+1)}\left(\begin{array}{l}
n \\
k
\end{array}\right)\left(\frac{c}{a}\right)^{\delta_{k}} \mathrm{E}\left[Y^{\delta_{k}} \psi(Y) \mid \mathcal{F}_{t}\right] .
$$

This result can be checked to follow essentially from the specialization of Theorem 5.6 to the case $N=0$. Briefly, its relevance is in that it reduces the computation of $\mathrm{E}\left[\rho(Y) \mid \mathcal{F}_{t}\right]$ 
to the computation of the scalar products $\left\langle\left(w_{\beta} / w_{\alpha}\right) \varphi_{c}, L_{n}^{\alpha}\right\rangle_{\alpha}$ and the $\psi$-twisted moments $\mathrm{E}\left[Y^{\kappa} \psi(Y) \mid \mathcal{F}_{t}\right]$. From Section 2.1, the scalar products are integrals of $\varphi_{c}$. Together with the $\psi$-twisted moments they may therefore be considered primitives of the problem.

\subsection{The second type of reduction series}

As a second step, this section addresses the construction of stochastic functionals as elements of Hilbert spaces using ladder height reduction series. For this we have to amend the setting of Section 6.1, which we adopt in this section.

For any positive integer $N$, we make the following three additional assumptions. First, for some, and thus any, positive real $c$, let $\varphi_{c}$ be $(N-1)$-fold continuously differentiable on $(L(c), \infty)$. Second, assume that the $(N-1)$ th derivative, $\varphi_{c}{ }^{(N-1)}$, is then a nonzero constant. Third, let $c$ be such that the inequalities

$$
0<\gamma_{A}\left(\varphi_{c}^{(\ell)}\right)+\ell+1+\frac{a}{c}\left(\gamma_{A}(\psi)+\gamma_{A}(f)\right), \quad A \in\{L(c), \infty\}
$$

hold for any $\ell$ in $\{0,1, \ldots, N-2\}$. Here $f \equiv f_{Y, t}$ is the density of $Y$ conditional on information at time $t$.

Our precise result, generalizing findings of Dufresne (2000), is then as follows.

Theorem 6.2. In the above setting, let the reals $\alpha>-1$ and $\beta$ satisfy the following two inequalities:

$$
1<2 \frac{a}{c}\left(\delta_{\infty}(\psi)+\delta_{\infty}(f)\right) \text { and } \max \{2 \beta-(2 N+\alpha+1),-2\}<2 \frac{a}{c}\left(\gamma_{0}(\psi)+\gamma_{0}(f)\right) .
$$

Then we have the ladder height reduction series representation

$$
\mathrm{E}\left[\rho(Y) \mid \mathcal{F}_{t}\right]=w_{\beta}(L(c)) \sum_{n=0}^{\infty} b_{n} L_{n}^{\alpha}(L(c)),
$$

which is the evaluation at the point $L(c)$ of the element $\sum_{n=0}^{\infty} b_{n} L_{n}^{\alpha}$ of the Hilbert space $L_{\alpha}^{2}(0, \infty)$ and, furthermore, is absolutely convergent as a series of complex numbers.

Here the coefficients $b_{n}$ depend on $\alpha, \beta, a, c$, and $N$ through linear combinations of $\psi$-twisted moments of $Y$, as follows:

$$
b_{n}=\sum_{k=0}^{n} b_{n, k} \mathrm{E}\left[Y^{N+\delta_{k}} \psi(Y) \mid \mathcal{F}_{t}\right]
$$

with

$$
b_{n, k}=\varphi_{c}^{(N-1)}\left(x_{0}\right) \frac{\alpha_{n, k}}{\left\|L_{n}^{\alpha}\right\|_{\alpha}^{2}} \chi\left(\frac{a}{c}\right) \frac{(c / a)^{N+\delta_{k}}}{\left(\delta_{k}+1\right)_{N}} \quad \text { and } \quad \delta_{k}=\alpha-\beta+k .
$$

Moreover, here $x_{0}$ is any element of $(L(c), \infty), w_{\beta}(x)=x^{\beta} \exp (-x)$, and $(\lambda)_{N}$ is the $N$ th Pochhammer symbol of any complex number $\lambda$, recursively defined by $(\lambda)_{0}=1$ and $(\lambda)_{N}=$ $(\lambda+N-1)(\lambda)_{N-1}$ for $N \geq 1$.

Proof of Theorem 6.2. We prove the theorem by specializing Theorem 5.5. In fact, the two inequalities displayed in Theorem 5.5 imply that the ladder height densities $\lambda_{0}\left(g_{c}\right), \ldots, \lambda_{N}\left(g_{c}\right)$ 
are well defined. By successively using the log-additivity of $\gamma_{A}$ (see Proposition 2.1), the permanence properties in Proposition 5.2, and Theorem 5.4, we then have

$$
\gamma_{A}\left(\varphi_{c}^{(\ell)} \lambda_{\ell+1}\left(g_{c}\right)\right)=\gamma_{A}\left(\varphi_{c}^{(\ell)}\right)+\gamma_{A}\left(g_{c}\right)+\ell+1 \geq \gamma_{A}\left(\varphi_{c}^{(\ell)}\right)+\ell+1+\frac{a}{c}\left(\gamma_{A}(\psi)+\gamma_{A}(f)\right) .
$$

The initial set of $2(N-1)$ inequalities in $\gamma_{A}$ thus imply that the limits of $\left(\varphi_{c}^{(\ell)} \lambda_{\ell+1}\left(g_{c}\right)\right)(x)$ as $x$ tends to $L(c)$ and to $\infty$ are equal to 0 for $\ell$ in $\{0,1, \ldots, N-2\}$. Hence, Corollary 4.2 gives

$$
\mathrm{E}\left[\rho(Y) \mid \mathcal{F}_{t}\right]=\varphi_{c}^{(N-1)}\left(x_{0}\right) a_{N-1}\left(g_{c}\right) \lambda_{N}\left(g_{c}\right)(L(c)),
$$

where

$$
a_{N-1}\left(g_{c}\right)=\chi\left(\frac{a}{c}\right) \frac{(c / a)^{N}}{N !} \mathrm{E}\left[Y^{N} \psi(Y) \mid \mathcal{F}_{t}\right] .
$$

Now substitute for $\lambda_{N}\left(g_{c}\right)(L(c))$ the expansion of Theorem 5.5, namely

$$
\lambda_{N}\left(g_{c}\right)(L(c))=w_{\beta}(L(c)) \sum_{n=0}^{\infty} b_{N, n} L_{n}^{\alpha}(L(c))
$$

with

$$
b_{N, n}=\sum_{k=0}^{n} \frac{\alpha_{n, k}}{\left\|L_{n}^{\alpha}\right\|_{\alpha}^{2}}\left(\frac{c}{a}\right)^{\delta_{k}} \frac{\mathrm{E}\left[Y^{N+\delta_{k}} \psi(Y) \mid \mathcal{F}_{t}\right]}{\mathrm{E}\left[Y^{N} \psi(Y) \mid \mathcal{F}_{t}\right]}\left(\begin{array}{c}
N+\delta_{k} \\
\delta_{k}
\end{array}\right)^{-1} .
$$

On collecting terms, the proof of Theorem 6.2 is complete.

\subsection{Constructing functionals from statistical data}

A principal raison d'être for developing ladder height reduction series seems to be their use not in computing stochastic functionals but rather in answering questions related to the vector space or Hilbert space structure of the dual of $L_{\alpha}^{2}(0, \infty)$. Ordering aspects of such functionals would furnish examples of such questions, but we want to stress methods for their actual construction here.

In briefly discussing this last theme, we assume both that the Section 6.1 function $L$ is the identity mapping and that the convergence of the Theorem 6.2 reduction series in $L_{\alpha}^{2}(0, \infty)$ is determined by the ratio $\theta=c / a$ ranging over some nonempty open subinterval of the positive reals. This is a situation typical in option valuation where the parameter $a$ can be interpreted as being, or as being related to, the strike price of a call option. In this picture it makes sense to think of the parameter $a$ as ranging over the whole positive real line, and to require at time $t$ an empirically observed function for $R_{t}(a \theta)=\mathrm{E}\left[\left(\psi \varphi_{a}\right)(Y) \mid \mathcal{F}_{t}\right]$. Assume that such a function can be constructed in $L_{\alpha}^{2}(0, \infty)$ with Laguerre expansion

$$
R_{t}(a \theta)=w_{\beta}(a \theta) \sum_{n=0}^{\infty} b_{n}^{*} L_{n}^{\alpha}(a \theta), \quad a \in(0, \infty) .
$$

Its Laguerre coefficients $b_{n}^{*}$ are thus determined from empirical data. To connect with this paper's approach, at this point now turn Theorem 6.2 into an ansatz by postulating the validity of the representation,

$$
R_{t}(a \theta)=w_{\beta}(a \theta) \sum_{n=0}^{\infty} b_{n} L_{n}^{\alpha}(a \theta), \quad a \in(0, \infty),
$$


given there. Since we have thus arrived at two representations of the same function in terms of the Hilbert basis of $L_{\alpha}^{2}(0, \infty)$ furnished by the $\alpha$-Laguerre polynomials $L_{n}^{\alpha}$, their coefficients must be equal. For any nonnegative integer $n$, we thus obtain, on comparison,

$$
b_{n}^{*}=\sum_{k=0}^{n} b_{n, k} \mathrm{E}\left[Y^{N+\delta_{k}} \psi(Y) \mid \mathcal{F}_{t}\right],
$$

where

$$
b_{n, k}=\varphi_{c}^{(N-1)}\left(x_{0}\right) \frac{\alpha_{n, k}}{\left\|L_{n}^{\alpha}\right\|_{\alpha}^{2}} \chi\left(\frac{1}{\theta}\right) \frac{\theta^{N+\delta_{k}}}{\left(\delta_{k}+1\right)_{N}}
$$

is nonzero for any $k$. Solving this system of equations determines the $\psi$-twisted moments of $Y$. These values can now be input to the Laguerre reduction series of Theorem 6.1 for numerical use, or, proceeding optimistically, used to determine the law of $Y$.

On a conceptual level, the basis for this is the ladder height density representation furnished by Corollary 4.2. More precisely, recall that this corollary states that the stochastic functionals under consideration are equal to a nonzero constant times the value of a ladder height density. Such ladder height representations extend to a multidimensional framework. In fact, if $g$ is the joint density of $n$ positive random variables, then the iterative construction of Section 3.1 extends to one of $k$ th ladder height densities $\lambda_{k}(g)$ on the positive orthant, $P_{+}=\mathbb{R}_{>0}^{n}$, of $\mathbb{R}^{n}$, as follows. Set $\lambda_{0}(g)=g$ and recursively define

$$
\lambda_{k+1}(g)(x)=\frac{1}{M_{1}\left[\lambda_{k}(g)\right]} \int_{P_{+}(x)} \lambda_{k}(g)(w) \mathrm{d} w
$$

for any $x$ in $P_{+}$if the first multimoment,

$$
M_{1}\left[\lambda_{k}(g)\right]=\int_{P_{+}} w_{1} \cdots w_{n} \lambda(g)(w) \mathrm{d} w,
$$

of $\lambda_{k}(g)$ is finite and positive. Here $P_{+}(x)$ is the translation of $P_{+}$by the vector $x$, i.e. $P_{+}(x)$ consists of all vectors $w=\left(w_{1}, \ldots, w_{n}\right)$ in $P_{+}$such that $w_{k}>x_{k}$ for all $k$. This construction preserves, in particular, any product structure of $g$. If $g$ is a product of functions of one positive variable, then the results of Sections 4 and 5 carry over verbatim to any $\lambda_{k}(g)$ when applied to each of its factors separately. In a general situation, using multidimensional Laguerre expansions as in Thangavelu (1993), extensions of the results suggest themselves, but care has to be taken to make them rigorous. We hope to be able to discuss all this in fuller detail in another publication.

\subsection{A summary with a view towards generality}

The concepts and constructions developed in this paper apply in greater generality, in part at the level of Banach space-valued random variables. While we have been seeking to give an exemplary discussion which can be adapted to the requisite needs of generality, it may be worthwhile to summarize one of the key notions of the approach, the reduction series, both from this point of view and from a slightly more general perspective.

Consider any random variable $Y$ with values in any subinterval $I=(a, b)$ of the reals. Then let $w$ be any positive function on $I$ which is continuous almost everywhere and for which the product, $w|q|$, of $w$ and the absolute value of any polynomial $q$ is integrable on $I$. Proceeding as in Section 2.1, we define $L_{w}^{2}(I)$ to be the space of all real-valued functions $F$ on $I$ which 
are $w$-square integrable, i.e. such that $\|F\|_{w}^{2}=\int_{I} w(x)|F(x)|^{2} \mathrm{~d} x<\infty$. The norm $\|\cdot\|_{w}$ is induced by the bilinear form $\langle F, G\rangle_{w}=\int_{I} w(x) F(x) G(x) \mathrm{d} x$, and when equipped with this form $L_{w}^{2}(I)$ becomes a Hilbert space. As such it has a Hilbert space basis, $\mathcal{P}$, by which we understand a maximal subset of pairwise-orthogonal, nonzero-norm elements of $L_{w}^{2}(I)$.

To demonstrate the analogues of the results of Section 6.2 in this setting, we make the necessary additional assumption that the quotient $g=f_{Y} / w$ of the density $f_{Y}$ of $Y$ divided by the weight $w$ is in $L_{w}^{2}(I)$. Hence, we have the representation

$$
g=\frac{f_{Y}}{w}=\sum_{p \in \mathcal{P}} a_{p} p
$$

with coefficients

$$
a_{p}=\frac{\langle p, g\rangle_{w}}{\langle p, p\rangle_{w}}=\frac{\mathrm{E}[p(Y)]}{\langle p, p\rangle_{w}},
$$

which is convergent in $w$-mean. By general theory, at most countably many of these coefficients are nonzero, and we let $\left\{p_{n}: n \in \mathbb{Z}_{n \geq 0}\right\}$ be an enumeration of a subset of $\mathcal{P}$ such that $a_{p}=0$ for all $p$ in its complement. Now define the $N$ th order error term, $r_{N}$, of this representation by

$$
r_{N}=\sum_{k=N}^{\infty} a_{p_{k}} p_{k},
$$

which is such that $\left\|r_{N}\right\|_{w}$ goes to 0 as $N$ tends to $\infty$. Proceeding along the lines of Section 6.2, for functionals of $Y$ associated with sufficiently nice functions $\rho$ on $I$ we thus obtain the representation

$$
\mathrm{E}[\rho(Y)]=\sum_{k=0}^{N-1} \mathrm{E}[p(Y)] \frac{\left\langle p_{k}, \rho\right\rangle_{w}}{\left\langle p_{k}, p_{k}\right\rangle_{w}}+R_{N},
$$

whose remainder terms, $R_{N}$, satisfy the estimate

$$
\left|R_{N}\right| \leq\|\rho\|_{w}\left\|r_{N}\right\|_{w}
$$

and will also converge to 0 as $N$ tends to $\infty$ if $\rho$ is in $L_{w}^{2}(I)$.

The second type of reduction series, from Section 6.3, can be similarly generalized to the present situation, in both cases, and a reduction thus occurs from studying general functionals $\mathrm{E}[\rho(Y)]$ to studying functionals $\mathrm{E}[p(Y)]$ associated with the Hilbert space basis elements $p$.

A crucial issue, however, is if this reduction increases the tractability of the problem. A test case is furnished by option valuation where $\rho$ is part of an affine linear map and the problems in computing $\mathrm{E}[\rho(Y)]$ originate principally in the irrationalities caused by the pertinent truncations of the density of $Y$. If now, for example, Bessel functions $J_{\alpha}$ turn out to be elements of $\mathcal{P}$, the above reduction entails a reduction to expectations $\mathrm{E}\left[J_{\alpha}(Y)\right]$. These will in general have more severe irrationalities than do the expectations in the original problem and, so, will in general be more difficult to understand and handle explicitly. To increase tractability, we would therefore have to require that the functions in $\mathcal{P}$ not tend to increase the severity of the irrationalities in the problems; polynomials should possess such properties to a high degree.

Asking for Hilbert space bases that consist of polynomials is equivalent to requiring that only the zero functional of $L_{w}^{2}(I)$ takes the value 0 on all polynomials. To test this, it is sufficient to require it on the monomials $q_{n}(x)=x^{n}$ with $n$ any nonnegative integer. Moreover, using the Riesz representation theorem, a reduction thus occurs to the following moment problem:

for any nonzero $F \in L_{w}^{2}(I)$, there is an index $n_{F}$ with $\left\langle q_{n_{F}}, F\right\rangle_{w} \neq 0$, 
or, equivalently,

$$
\left\langle q_{n_{F}}, F\right\rangle_{w}=0 \text { for all } n \in \mathbb{Z}_{\geq 0} \text { only if } F=0 .
$$

If this moment problem holds for $w$, Gram-Schmidt orthogonalization of the $q_{n}$ will produce a basis $\mathcal{P}$ with the desired properties. With counterexamples furnished by many standard choices of $w$, this is, however, a restrictive condition. It may be argued that it is sufficient to have the density quotients $f_{Y} / w$ in the closure of the polynomials. This, however, reduces to constructing this closure in $L_{w}^{2}(I)$ and, thus, to solving a possibly more difficult problem. In this way, the study of stochastic functionals finally relates to a classical problem: the construction of 'good' orthogonal polynomials.

\section{Acknowledgements}

The hospitality and support of the Mathematics Department of ETH, Zürich, in writing this paper, as well as partial support by the Deutsche Forschungsgemeinschaft via a project with Christian Hipp (Universität Karlsruhe (TH)), are gratefully acknowledged. Thanks go to the referee for comments and a careful reading of the text.

\section{References}

ANH, V. V. AND LeOnEnKo, N. N. (1999). Non-Gaussian scenarios for the heat equation with singular initial conditions. Stoch. Process. Appl. 84, 91-114.

Apostol, T. (1974). Mathematical Analysis, 2nd edn. Addison-Wesley, Reading, MA.

Dufresne, D. (2000). Laguerre series for Asian and other options. Math. Finance 10, 407-428.

Dunford, N. ANd Schwartz, J. T. (1957). Linear Operators. Part I. John Wiley, New York.

Erdélyi, A., Magnus, W., Oberhettinger, F. and Tricomi, F. G. (1953). Higher Transcendental Functions, Vol. 2. McGraw-Hill, New York.

KatZnelson, Y. (1976). An Introduction to Harmonic Analysis, 2nd edn. Dover, New York.

Lebedev, N. N. (1972). Special Functions and Their Applications. Dover, New York.

Press, W. H., Teukolsky, S. A., Vetterling, W. T. and Flannery, B. P. (1992). Numerical Recipes in C, 2nd edn. Cambridge University Press.

Sansone, G. (1991). Orthogonal Functions. Dover, New York.

SCHRÖDER, M. (2005). Laguerre series in contingent claim valuation, with applications to Asian options. Math. Finance 15, 491-531.

Schröder, M. (2006). On ladder height densities and Laguerre series in the study of stochastic functionals. II. Exponential functionals of Brownian motion and Asian option values. Adv. Appl. Prob. 38, 995-1027.

Thangavelu, S. (1993). Lectures on Hermite and Laguerre Expansions. Princeton University Press. 